\title{
Structure formation during the collapse of a dipolar atomic Bose-Einstein condensate
}

\author{
N. G. Parker, ${ }^{1}$ C. Ticknor, ${ }^{2}$ A. M. Martin, ${ }^{3}$ and D. H. J. O’Dell ${ }^{1}$ \\ ${ }^{1}$ Department of Physics and Astronomy, McMaster University, Hamilton, Ontario, L8S 4M1, Canada \\ ${ }^{2}$ ARC Centre of Excellence for Quantum-Atom Optics and Centre for Atom Optics and Ultrafast Spectroscopy, \\ Swinburne University of Technology, Hawthorn, Victoria 3122, Australia \\ ${ }^{3}$ School of Physics, University of Melbourne, Parkville, Victoria 3010, Australia
}

(Received 11 October 2008; published 21 January 2009)

\begin{abstract}
We investigate the collapse of a trapped dipolar Bose-Einstein condensate. This is performed by numerical simulations of the Gross-Pitaevskii equation and the novel application of the Thomas-Fermi hydrodynamic equations to collapse. We observe regimes of both global collapse, where the system evolves to a highly elongated or flattened state depending on the sign of the dipolar interaction, and local collapse, which arises due to dynamically unstable phonon modes and leads to a periodic arrangement of density shells, disks, or stripes. In the adiabatic regime, where ground states are followed, collapse can occur globally or locally, while in the nonadiabatic regime, where collapse is initiated suddenly, local collapse commonly occurs. We analyze the dependence on the dipolar interactions and trap geometry, the length and time scales for collapse, and relate our findings to recent experiments.
\end{abstract}

DOI: $10.1103 /$ PhysRevA.79.013617

PACS number(s): 03.75.Kk, 75.80.+q

Wave packet collapse is a phenomenon seen in diverse physical systems whose common feature is that they obey nonlinear wave equations [1], e.g., in nonlinear optics [2], plasmas [3], and trapped atomic Bose-Einstein condensates (BECs) [4-9]. In the latter case, collapse occurs when the atomic interactions are sufficiently attractive. For the usual case of isotropic $s$-wave interactions experiments have demonstrated both global [5] and local collapse [7] depending upon, respectively, whether the imaginary healing length is of similar size or much smaller than the BEC [10]. During global collapse the monopole mode becomes dynamically unstable and the BEC evolves towards a point singularity, with the threshold for collapse generally exhibiting a weak dependence on trap geometry $[11,12]$. Local collapse occurs when a phonon mode is dynamically unstable such that the collapse length scale is considerably smaller than the BEC.

Recently, the Stuttgart group demonstrated collapse in a BEC with dipole-dipole interactions, where the atomic dipoles were polarized in a common direction by an external field $[8,9]$. The long-range nature of dipolar interactions means that the Gross-Pitaevskii wave equation that governs the BEC is not only nonlinear but also nonlocal [13-16]. On top of being long range, dipolar interactions are also anisotropic, being attractive in certain directions and repulsive in others. This anisotropy has manifested itself experimentally in the stability of the ground state, which is strongly dependent on the trap geometry [8], and in the anisotropic collapse of the condensate [9]. Some uncertainty exists over the mechanism of collapse in these systems. In the latter experiment, striking images indicate that the condensate underwent global collapse, which is likely to have occurred through a quadrupole mode [16-18]. In the former experiment, however, recent theoretical results suggest that local collapse played a role [19].

A unique feature of trapped dipolar BECs in comparison to $s$-wave BECs is that they are predicted to exhibit minima in their excitation spectrum at finite values of the excitation quantum number [20-25], reminiscent of the roton minimum in the quantum liquid He-II. For gaseous BECs the depth of the minimum is tunable via microscopic parameters such as the dipolar and $s$-wave interaction strengths. An important physical consequence of the "roton" minimum is that it can lead to density modulations in the ground-state dipolar BEC $[20,22,24-28]$. However, the regions of parameter space where they occur are small and lie close to the unstable region $[24,28]$. It is therefore natural to ask if these characteristic density modulations form during collapse where they might be more readily visible. As we shall see, collapse experiments can indeed provide us with an indirect yet accessible way of studying such effects.

The dipolar BECs that have been realized thus far $[29,30]$ are formed of ${ }^{52} \mathrm{Cr}$ atoms with magnetic dipole moments $d$ and coupling strength $C_{d d}=\mu_{0} d^{2}$, where $\mu_{0}$ is the permeability of free space. Ultracold quantum gases of polar molecules, which feature electric dipolar interactions, are also likely to be formed in the near future [31]. The dipolar interactions typically coexist with $s$-wave interactions of characteristic amplitude $g=4 \pi \hbar^{2} a_{s} / m$, where $a_{s}$ is the $s$-wave scattering length, and an important parameter is the ratio [32]

$$
\varepsilon_{d d}=C_{d d} / 3 g .
$$

The $s$-wave coupling $g$ can be effectively tuned between positive and negative infinity using a Feshbach resonance, and this was employed to control the stability of the dipolar BEC in the recent experiments $[8,9]$. Both the amplitude and sign of the dipolar coupling $C_{d d}$ can also be tuned by rotation of the polarization axis [33]. A huge parameter space of dipolar interactions, from $\varepsilon_{d d}=-\infty$ to $+\infty$, is thereby accessible for study.

Insight into the collapse instability of a trapped dipolar BEC can be obtained by considering its ground states. Such a route could be followed by beginning with a stable ground state and adiabatically tuning the parameters towards the instability, which we term "adiabatic collapse." Consider tuning the ratio $\varepsilon_{d d}$ : as it is increased the ground-state density profile evolves so as to benefit from the attractive part of the interaction. This can happen both globally and locally. For 
example, when $C_{d d}>0$ the system can undergo global magnetostriction and elongate along the axis of polarization $[13,15,16,34,35]$, tending towards a collapsed state of a line of end-to-end dipoles. However, the dipoles can also rearrange themselves locally $[20,22,24-28]$ and, in particular, in a pancake-shaped geometry for $C_{d d}>0$ a "red blood cell" density profile has been predicted [24].

If collapse is triggered suddenly, which we term nonadiabatic collapse, the system does not follow the ground state solutions and excitations play a role. In the dipolar collapse experiment [9] the collapse was initiated by a change in $g$ which took place over approximately one trap period. This time scale lies on the border between adiabatic and nonadiabatic collapse.

Motivated by these issues we study theoretically the global and local collapse of a dipolar BEC over a significant range of $\varepsilon_{d d}$ that is accessible to current experiments. Our analysis is based on simulations of the Gross-Pitaevskii equation and the hydrodynamic (Thomas-Fermi) approximation, including the novel application of the hydrodynamic equations of motion to collapse. We observe that collapse occurs anisotropically with the dipoles tending to align themselves end-to-end for $C_{d d}>0$ and side-by-side for $C_{d d}<0$. If collapse is approached adiabatically it occurs globally for moderate dipolar interactions $\left(-1 \leqq \varepsilon_{d d} \lesssim 2\right)$ and beyond this we have also observed signs of local collapse. When collapse is initiated suddenly, it is dominated by the formation of local density structures, whose shape is determined by the dipolar interactions and can be related to unstable Bogoliubov modes. We map out the length and time scales for collapse, and the role of interaction strength and trap geometry. We then compare our results to recent experiments, in particular, that of Lahaye et al. [9], where the condensate appeared to undergo global collapse. We show that our results are consistent with this observation and, furthermore, indicate how local collapse could be induced in this current experimental setup.

In Sec. I we introduce the mean-field description of dipolar BECs and, by employing the Thomas-Fermi approximation, derive the static solutions of the system and hydrodynamic equations of motion. In Sec. II we discuss the static solutions and their threshold for collapse. In Sec. III we consider nonadiabatic collapse, induced by a sudden change in the interaction strength, and compare the hydrodynamic predictions with simulations of the Gross-Pitaevskii equation. In Sec. IV, we extend our analysis of nonadiabatic collapse to map out the time and length scales of collapse, and in the latter case, show that the homogeneous Bogoliubov spectrum gives good agreement with the observed local collapse. In Sec. V we relate our findings to recent collapse experiments $[8,9]$ and in Sec. VI we present our conclusions.

\section{THEORETICAL FRAMEWORK}

\section{A. Dipolar Gross-Pitaevskii equation}

For weak interactions and at zero temperature the meanfield "wave function" of an atomic BEC $\psi \equiv \psi(\mathbf{r}, t)$ satisfies the Gross-Pitaevskii equation (GPE)

$$
i \hbar \frac{\partial \psi}{\partial t}=\left(-\frac{\hbar^{2}}{2 m} \nabla^{2}+V(\mathbf{r})+g|\psi|^{2}+\Phi_{d d}(\mathbf{r}, t)\right) \psi
$$

We assume that the confining potential has the cylindrically symmetric harmonic form

$$
V_{\text {ext }}(\mathbf{r})=\frac{1}{2} m \omega_{x}^{2}\left(x^{2}+y^{2}+\gamma^{2} z^{2}\right),
$$

where $\omega_{x}$ is the radial trap frequency and $\gamma=\omega_{z} / \omega_{x}$ is the trap's aspect ratio (which will be henceforth termed the trap ratio). Note that the trap has axial and radial harmonic oscillator lengths $a_{z}=\sqrt{\hbar / m \omega_{z}}$ and $a_{x}=\sqrt{\hbar / m \omega_{x}}$. Dipolar atomic interactions are described by the nonlocal mean-field potential $\Phi_{d d}[13-15,37]$

$$
\Phi_{d d}(\mathbf{r})=\int d^{3} \mathbf{r}^{\prime} U_{d d}\left(\mathbf{r}-\mathbf{r}^{\prime}\right)\left|\psi\left(\mathbf{r}^{\prime}\right)\right|^{2} .
$$

The interaction potential between two dipoles separated by $\mathbf{r}$ and aligned by an external field along a unit vector $\hat{\mathbf{e}}$ is given by

$$
U_{d d}(\mathbf{r})=\frac{C_{d d}}{4 \pi} \hat{e}_{i} \hat{e}_{j} \frac{\left(\delta_{i j}-3 \hat{r}_{i} \hat{r}_{j}\right)}{r^{3}} .
$$

Throughout this work we consider the dipoles to be aligned in the $z$ direction. It is useful to specify the dipolar interaction strength in Eq. (2) by the parameter

$$
k_{d d}=N a_{d d} / a_{x},
$$

where $a_{d d}=C_{d d} m /\left(12 \pi \hbar^{2}\right)$ is the dipolar "scattering length" and $N$ is the total number of condensate atoms. Note that the ratio $\varepsilon_{d d}$ can be written as $\varepsilon_{d d}=a_{d d} / a_{s}$. In the dipolar BEC collapse experiments $[8,9] k_{d d}$ lies in the range $25-50$. We will assume throughout this work that $g>0$ such that the case of $\varepsilon_{d d}<0$ corresponds to $C_{d d}<0$. While the opposing case of $g<0$ is experimentally accessible, the $s$-wave interactions will typically induce collapse, rather than the dipolar interactions, and so will not be considered in this work.

It is important to note that the basic GPE is insufficient to describe the full collapse dynamics since higher-order effects, e.g., three-body loss, can become significant as the density escalates. However, the GPE provides an excellent prediction for the onset of collapse $[8,11,12]$ and can be expected to accurately describe the early collapse dynamics. Our study will therefore consider the dynamics up to this point. Extension of these results to the full collapse dynamics could be made in future by including a three-body loss term in the GPE $[9,18]$.

\section{B. Thomas-Fermi solutions}

Static solutions of the GPE can be expressed as $\psi(\mathbf{r}, t)$ $=\psi_{0}(\mathbf{r}) \exp [-i \mu t / \hbar]$, where $\mu$ is the chemical potential. We enter the Thomas-Fermi (TF) regime when the interaction energy dominates over the energy arising from density gradients, termed the zero-point kinetic energy [41]. We then neglect the zero-point energy and the atomic density $n_{0}$ $=\left|\psi_{0}\right|^{2}$ satisfies the expression

$$
V(\mathbf{r})+g n_{0}(\mathbf{r})+\Phi_{d d}(\mathbf{r})=\mu .
$$


For an $s$-wave BEC, the ratio of interaction energy to zero-point energy is commonly specified as $k_{\mathrm{s}}=N a_{\mathrm{s}} / a_{x}$, with the system entering the TF regime when $k_{\mathrm{s}} \gg 1$. The criterion for the TF regime is not so simple for a dipolar BEC since the anisotropic interactions make it strongly dependent on its shape. However, in the limiting cases of a highly elongated "cigar" condensate, or a highly flattened "pancake" condensate, the TF regime is valid when, respectively [36],

$$
\begin{gathered}
\frac{\gamma k_{d d}}{\varepsilon_{d d}}\left(1-\varepsilon_{d d}\right) \gg 1 \text { (cigar), } \\
\frac{k_{d d}}{\gamma^{3 / 2} \varepsilon_{d d}}\left(1+2 \varepsilon_{d d}\right) \gg 1 \text { (pancake). }
\end{gathered}
$$

A solution of Eq. (7) is given by an inverted parabola of the form [34]

$$
n_{0}(\mathbf{r})=\frac{15 N}{8 \pi R_{x 0} R_{y 0} R_{z 0}}\left[1-\frac{x^{2}}{R_{x 0}^{2}}-\frac{y^{2}}{R_{y 0}^{2}}-\frac{z^{2}}{R_{z 0}^{2}}\right],
$$

valid where $n_{0}(\mathbf{r}) \geqslant 0$ and $n_{0}(\mathbf{r})=0$ elsewhere, and the TF radii of the condensate are denoted by $R_{x 0}, R_{y 0}$, and $R_{z 0}$. Note that the subscript 0 denotes static solutions. When the trap is cylindrically symmetric about the same direction as the polarizing field, as we assume here, the density is also cylindrically symmetric. Its aspect ratio $\kappa_{0}=R_{x 0} / R_{z 0}$ satisfies a transcendental equation $[16,34]$

$$
\frac{\kappa_{0}^{2}}{\gamma^{2}}\left[\frac{3 \varepsilon_{d d} f\left(\kappa_{0}\right)}{1-\kappa_{0}^{2}}\left(\frac{\gamma^{2}}{2}+1\right)-2 \varepsilon_{d d}-1\right]=\varepsilon_{d d}-1,
$$

where

$$
f(\kappa)=\frac{1+2 \kappa^{2}}{1-\kappa^{2}}-\frac{3 \kappa^{2} \operatorname{arctanh} \sqrt{1-\kappa^{2}}}{\left(1-\kappa^{2}\right)^{3 / 2}} .
$$

The equilibrium radii are given by $[34]$

$$
R_{x 0}=R_{y 0}=\left\{\frac{15 N g \kappa_{0}}{4 \pi m \omega_{x}^{2}}\left[1+\varepsilon_{d d}\left(\frac{3}{2} \frac{\kappa_{0}^{2} f\left(\kappa_{0}\right)}{1-\kappa_{0}^{2}}-1\right)\right]\right\}^{1 / 5}
$$

and $R_{z 0}=R_{x 0} / \kappa_{0}$. For an $s$-wave BEC, we retrieve the simple and expected result that $\kappa_{0}=\gamma$. The anisotropic interactions, however, lead to magnetostriction such that $\kappa_{0}<\gamma$ for $\varepsilon_{d d}$ $>0$ and $\kappa_{0}>\gamma$ for $\varepsilon_{d d}<0$.

Consider the broader range of cylindrically symmetric density profiles which have the same form as Eq. (10) but are not limited to the equilibrium solutions. These general profiles define an energy "landscape" in terms of $R_{x}$ and $\kappa$ given by $[35]$

$$
\frac{E\left(R_{x}, \kappa\right)}{N}=\frac{m \omega_{x}^{2} R_{x}^{2}}{14}\left(2+\frac{\gamma^{2}}{\kappa^{2}}\right)+\frac{15 N g \kappa}{28 \pi R_{x}^{3}}\left[1-\varepsilon_{d d} f(\kappa)\right] .
$$

Then the static solutions (11) correspond to stationary points in this landscape located at $\left(R_{x 0}, \kappa_{0}\right)$. By examining the landscape in the vicinity of the solution one can determine whether it is stable (a global or local energy minimum) or unstable (maximum or saddle point).
The TF regime can be formally regarded as the $N \rightarrow \infty$ limit of Gross-Pitaevskii theory. Indeed, the TF predictions for the stability of a dipolar BEC depend only upon $\varepsilon_{d d}$ and $\gamma$, thus simplifying the parameter space. However, the TF model does not accurately describe situations where the zeropoint energy is considerable, e.g., close to the collapse threshold. In order to discern the effect of the zero-point energy we shall compare the TF solutions with those of a variational approach based upon a gaussian ansatz, as detailed in Appendix A, which includes this energy contribution $[15,16]$. To describe deviations from the TF (or Gaussian) solutions, e.g., "red-blood cell" states [24], one must solve the full GPE. This will also be considered in due course.

\section{Thomas-Fermi equations of motion for collapse}

To enable a hydrodynamic interpretation we employ the Madelung transform $\psi(\mathbf{r}, t)=\sqrt{n(\mathbf{r}, t)} \exp [i S(\mathbf{r}, t)]$, where $n(\mathbf{r}, t)$ and $S(\mathbf{r}, t)$ are the density and phase distributions, and define the "fluid" velocity as $\mathbf{v}(\mathbf{r}, t)=(\hbar / m) \boldsymbol{\nabla} S(\mathbf{r}, t)$ [38]. In the TF limit the dipolar GPE then leads to hydrodynamic equations [41]

$$
\begin{gathered}
\frac{\partial n}{\partial t}=-\nabla \cdot(n \mathbf{v}), \\
m \frac{\partial \mathbf{v}}{\partial t}=-\nabla\left(\frac{m v^{2}}{2}+V+g n+\Phi_{d d}\right) .
\end{gathered}
$$

Following earlier work for $s$-wave BECs $[39,40]$ there exists a class of exact time-dependent scaling solutions to Eqs. (15) and (16) given by [34]

$$
\begin{aligned}
& n(\mathbf{r}, t)=n_{0}(t)\left[1-\frac{x^{2}}{R_{x}^{2}(t)}-\frac{y^{2}}{R_{y}^{2}(t)}-\frac{z^{2}}{R_{z}^{2}(t)}\right], \\
& \mathbf{v}(\mathbf{r}, t)=\frac{1}{2} \boldsymbol{\nabla}\left[\alpha_{x}(t) x^{2}+\alpha_{y}(t) y^{2}+\alpha_{z}(t) z^{2}\right]
\end{aligned}
$$

valid where $n(\mathbf{r}, t) \geqslant 0$ and $n(\mathbf{r}, t)=0$ elsewhere, and $n_{0}(t)$ $=15 N /\left[8 \pi R_{x}(t) R_{y}(t) R_{z}(t)\right]$ is the peak density. The time evolution of the radii $R_{j}$ is governed by three ordinary differential equations, with the components of the velocity field given by $\alpha_{j}=\dot{R}_{j} / R_{j}$, where $j=x, y, z$.

Restricting ourselves to cylindrically symmetric dynamics where $R_{x}(t)=R_{y}(t)$, and introducing the scaling factors $\lambda_{x}(t)$ $=R_{x}(t) / R_{x 0}$ and $\lambda_{z}(t)=R_{z}(t) / R_{z 0}$ (recall that the 0 subscript denotes the initial static solution), the time evolution is determined by the coupled ordinary differential equations

$$
\ddot{\lambda_{x}}=-\omega_{x}^{2} \lambda_{x}+\frac{\eta g(t) \kappa_{0}}{\lambda_{x} \lambda_{z}}\left[\frac{1}{\lambda_{x}^{2}}-\varepsilon_{d d}(t)\left(\frac{1}{\lambda_{x}^{2}}+\frac{3}{2} \kappa_{0}^{2} \frac{f\left(\kappa_{0} \lambda_{x} / \lambda_{z}\right)}{\kappa_{0}^{2} \lambda_{x}^{2}-\lambda_{z}^{2}}\right)\right],
$$



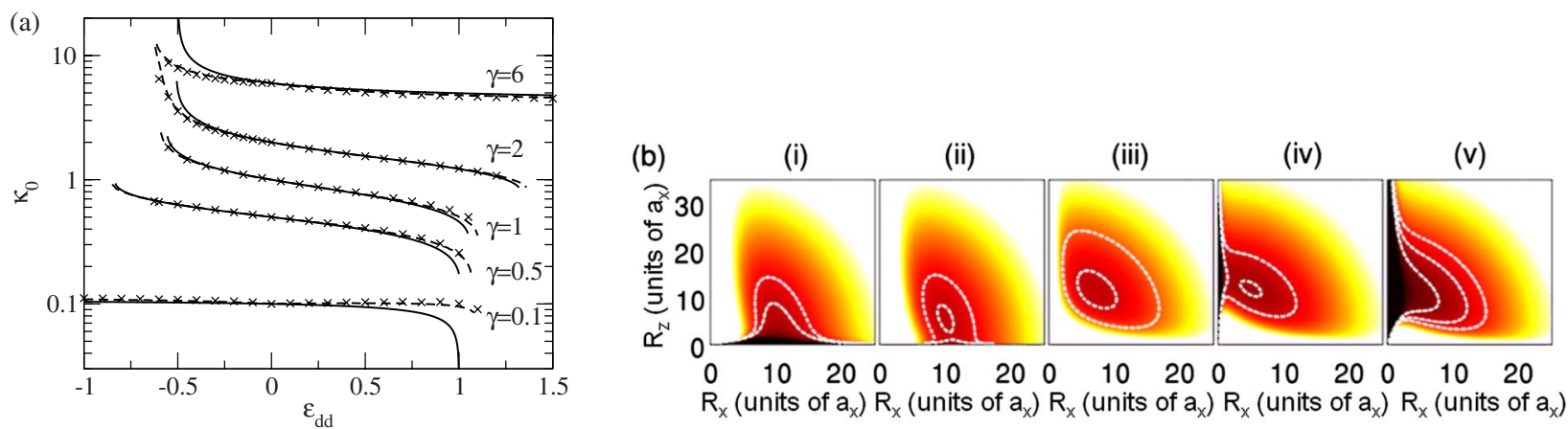

FIG. 1. (Color online) (a) Aspect ratio $\kappa_{0}$ of the ground state solutions as a function of $\varepsilon_{d d}$ for various trap ratios $\gamma$. Presented are the stable TF solutions of Eq. (11) (solid lines), GPE solutions with $k_{d d}=115$ (crosses), and variational solutions for $k_{d d}=115$ (dashed line). (b) Energy landscapes of Eq. (14) for $\gamma=2$ and $\varepsilon_{d d}=$ (i) -0.8 , (ii) -0.52 , (iii) 0.8 , (iv) 1.025, and (v) 1.4. Light (dark) regions correspond to high (low) energy, and white contours help to visualize the landscapes.

$\ddot{\lambda}_{z}=-\omega_{x}^{2} \gamma^{2} \lambda_{z}+\frac{\eta g(t) \kappa_{0}^{3}}{\lambda_{x}^{2}}\left[\frac{1}{\lambda_{z}^{2}}+2 \varepsilon_{d d}(t)\left(\frac{1}{\lambda_{z}^{2}}+\frac{3}{2} \frac{f\left(\kappa_{0} \lambda_{x} / \lambda_{z}\right)}{\kappa_{0}^{2} \lambda_{x}^{2}-\lambda_{z}^{2}}\right)\right]$

where $\eta=15 N / 4 \pi m R_{x 0}^{5}$. These equations are significantly less demanding to solve than the full GPE and in certain limits analytic solutions exist [see Eq. (26) below]. The TF equations of motion have been successfully applied to model the condensate dynamics under time-dependent trapping including the important case of ballistic expansion [41,42]. They describe two independent collective excitation modes of the system: the monopole mode [when $\lambda_{x}(t)$ and $\lambda_{z}(t)$ are in phase] and the axis-symmetric quadrupole mode [when $\lambda_{x}(t)$ and $\lambda_{z}(t)$ are $180^{\circ}$ out of phase]. In this paper we employ the TF equations of motion to study global collapse. In the pure $s$-wave case global collapse occurs through the monopole mode [10], but when dipolar interactions dominate global collapse occurs through the quadrupole mode [16-18].

We will employ the TF equations of motion to describe nonadiabatic collapse, triggered by a sudden change in $\varepsilon_{d d}(t)$. To conform to the current experimental method [8,9] we shall implement this through a sudden change in the $s$-wave interactions $g(t)$. By, (i) starting with the BEC well below the threshold for collapse and (ii) suddenly changing to a state which is well above the threshold for collapse, the regime where zero-point energy dominates can be by-passed and the TF equations should apply throughout. We will verify this in due course.

\section{ADIABATIC COLLAPSE}

Imagine an experiment that starts with a stable ground state BEC and adiabatically increasing the magnitude of $\varepsilon_{d d}$. The condensate will remain in the ground state corresponding to the instantaneous value of $\varepsilon_{d d}$ and will eventually collapse at a critical value of $\varepsilon_{d d}[13,15]$. The threshold for collapse in general depends upon $\varepsilon_{d d}, g, \gamma$, and $N$. We consider two possible scenarios for adiabatic collapse: (i) if there is no roton minimum in the excitation spectrum then adiabatic collapse proceeds in a similar manner to the usual pure $s$-wave case, i.e., a global collapse via a low-lying shape oscillation mode once the (imaginary) healing length becomes of the same order as the condensate size [10]; (ii) if there is a roton minimum then this deepens as $\varepsilon_{d d}$ increases and, at the point at which it reaches zero energy, can lead to local collapse on a length scale determined by the roton minimum. We note that technically speaking neither scenario can be truly adiabatic since the mode responsible for collapse has zero frequency at the collapse threshold, but the nonadiabaticity can be confined to a small region of parameter space if $\varepsilon_{d d}$ is increased slowly enough.

\section{A. Global adiabatic collapse}

Ground-state solutions, characterized by their aspect ratio $\kappa_{0}$, are presented in Fig. 1(a) as a function of $\varepsilon_{d d}$ for various trap ratios $\gamma$. The figure shows the predictions given by numerical solution of the GPE, the parabolic TF solution, and the Gaussian variational ansatz. We first consider the parabolic TF solutions [solid lines in Fig. 1(a)], which, we recall, can be characterized solely by $\varepsilon_{d d}$ and $\gamma$. For $\varepsilon_{d d}=0$ we observe that $\kappa_{0}=\gamma$, as expected for $s$-wave condensates. We will consider the regimes of positive and negative $\varepsilon_{d d}$ separately with the aid of typical energy landscapes pictured in Fig. 1(b)

$\varepsilon_{d d}>0$ : As $\varepsilon_{d d}$ is increased from zero, $\kappa_{0}$ decreases since the dipoles prefer to lie end-to-end along $z$. The experiment [44] observed this magnetostrictive effect, in good agreement with the TF predictions. For $0<\varepsilon_{d d}<1$, the parabolic solutions (10) are global minima of the TF energy functional [35] [Fig. 1(b)(iii)]. For $\varepsilon_{d d}>1$, however, the TF solution becomes only a local minimum [Fig. 1(b)(iv)], with the global minimum being a collapsed state of zero width. Indeed, there is an upper critical dipolar-to-s-wave ratio $\varepsilon_{d d}^{c+} \geqslant 1$, beyond which the local minimum disappears and the whole system is unstable to collapse into a $\kappa_{0}=0$ state [Fig. 1(b)(v)], i.e., an infinitely thin line of dipoles. Note that $\varepsilon_{d d}^{c+}$ depends on the trap ratio $\gamma$. Elongated BECs, being predominantly attractive, are most unstable, with $\varepsilon_{d d}^{c+} \approx 1$. Increasing $\gamma$ increases $\varepsilon_{d d}^{c+}$ due to the increasing repulsive interactions in the system. Indeed, for $\gamma \geqslant 5.17, \varepsilon_{d d}^{c+}=\infty[15,16,35]$, i.e., the parabolic solutions are robust to collapse for any interaction strength [see the case of $\gamma=6$ in Fig. 1(a)]. 
$\varepsilon_{d d}<0$ : As $\varepsilon_{d d}$ is decreased from zero, $\kappa_{0}$ increases since the dipoles now prefer to lie side-by-side in the transverse plane. For $-0.5<\varepsilon_{d d}<0$, the parabolic solutions are robust to collapse, while for $\varepsilon_{d d}<-0.5$, they become local energy minima with the global minima being a collapsed plane of dipoles [Fig. 1(b)(ii)]. We define a critical value $\varepsilon_{d d}^{c-} \leqslant-0.5$, below which no stable parabolic solutions exist [Fig. 1(b)(i)] and the system is unstable to collapse into a $\kappa_{0}=\infty$ state, i.e., an infinitely thin plane of side-by-side dipoles. Pancake geometries are particularly prone to this with $\varepsilon_{d d}^{c-} \approx-0.5$, while the system becomes increasingly stable in more elongated geometries. Indeed, in sufficiently elongated geometries $\gamma$ $\lesssim 0.19$, the parabolic solutions are stable to collapse with $\varepsilon_{d d}^{c-}=-\infty$.

Numerical solutions of the time-independent GPE for $k_{d d}=115$ are shown in Fig. 1(a) as crosses. Our method of determining the BEC widths is detailed in Appendix B. While the GPE solutions are generally in good agreement with the TF results, deviations become significant near the point of collapse where the zero-point energy has a considerable stabilizing effect on the solutions. We have additionally performed time-dependent simulations in which $\varepsilon_{d d}$ is varied slowly, and find that the condensate follows these ground-state solutions and maintains a paraboliclike density profile. This is consistent with global collapse. We have performed a similar analysis for $k_{d d}=14$ and observe the same qualitative results, with the threshold for collapse pushed to slightly higher values of $\left|\varepsilon_{d d}\right|$ due to enhanced zero-point energy.

The dashed lines in Fig. 1(a) are the results given by the Gaussian variational method of Appendix A. Note that if zero-point effects were neglected, the Gaussian solutions would satisfy the same transcendental equation (11) as the parabola, i.e., the aspect ratio of the BEC $\kappa$ is largely independent of the ansatz (although the profile and radii do differ). Indeed, for small values of $\left|\varepsilon_{d d}\right|$ the variational and TF predictions are almost identical. However, close to the onset of collapse these predictions deviate. Importantly, the gaussian variational method gives excellent agreement with the full GPE solutions right up to the point of collapse, highlighting the importance of zero-point energy at the point of collapse.

\section{B. Local adiabatic collapse}

A surprising result of the parabolic density profiles is that within the TF approximation they remain stable even as $\varepsilon_{d d} \rightarrow \infty$ and $\varepsilon_{d d} \rightarrow-\infty$, providing the trap ratio is sufficiently extreme $\left(\gamma>5.17\right.$ for $\varepsilon_{d d}>0$ and $\gamma<0.19$ for $\left.\varepsilon_{d d}<0\right)$. Similar behavior arises for a variational approach based on a Gaussian density profile $[15,16]$. The recent experiment of Koch et al. [8] has observed the stability of a dipolar condensate under various trap ratios, in reasonable qualitative agreement with the Gaussian and TF predictions. However, numerical solutions of the GPE show that, even though the stability is enhanced under extreme trap ratios, collapse will occur for strong enough dipolar interactions [16]. This apparent contradiction arises because the Gaussian and parabolic solutions are only capable of describing low-lying monopole and quadrupole fluctuations. In the presence of a roton minimum the instability of a mode with high quantum number can lead to local adiabatic collapse. Bohn et al. recently employed a theoretical model that allowed for local collapse and found improved quantitative agreement with the experimental observations in pancake geometries [19], suggesting that local collapse does indeed play a key role in such geometries. Note that, by contrast, in $s$-wave condensates an adiabatic reduction in $g$ should always induce global collapse.

In order to induce local adiabatic collapse it is necessary to use values of $\varepsilon_{d d}$ that fall outside $-1 \lesssim \varepsilon_{d d} \lesssim 2$ (the region of Fig. 1). We have probed pancake-shaped ground state solutions that extend beyond this range. For $\gamma=10$ and 20 we have probed up to $\varepsilon_{d d}=30$ with no evidence for collapse. However, for $\gamma=6$ we have observed the adiabatic onset of a local collapse instability at $\varepsilon_{d d} \approx 2.2$, characterized by the formation and growth of cylindrical density shells (similar to those that will be discussed in Sec. III). Our results are consistent with those of Ronen et al. [24], who predicted that, in a purely dipolar BEC $\left(\varepsilon_{d d}=\infty\right)$, collapse is possible for large $\gamma$ and that, close to the collapse threshold, the ground state adopts density corrugations. It is reasonable to assume that as one passes into the unstable region that these lead to local collapse. This picture was also recently suggested by Bohn et al. [19]. Note that for the moderate range of $\varepsilon_{d d}$ that we concentrate on here, the TF solutions are a very good approximation to the Gross-Pitaevskii solutions and the adiabatic collapse proceeds globally.

\section{NONADIABATIC COLLAPSE}

An alternative way to induce collapse is to perform a nonadiabatic change of $\varepsilon_{d d}$ of the form

$$
\varepsilon_{d d}= \begin{cases}\varepsilon_{d d}^{0} & \text { for } t=0, \\ \varepsilon_{d d}^{f} & \text { for } t>0 .\end{cases}
$$

We will assume that this is achieved by tuning the $s$-wave interactions from $g_{0}=C_{d d} / 3 \varepsilon_{d d}^{0}$ to $g_{f}=C_{d d} / 3 \varepsilon_{d d}^{f}$.

In order for the $\mathrm{TF}$ equations of motion to remain valid we require that the TF approximation holds throughout the dynamics, i.e., regimes where the zero-point energy is important are avoided. This requires that the initial condensate is well inside the regime of stable solutions $\left(\left|\varepsilon_{d d}^{0}\right|<\left|\varepsilon_{d d}^{c}\right|\right)$, and subsequently that the system is deep within the collapse regime $\left(\left|\varepsilon_{d d}^{f}\right|>\left|\varepsilon_{d d}^{c}\right|\right)$.

\section{A. Nonadiabatic collapse for $\varepsilon_{d d}^{f}>0$}

We begin with a stable condensate with $\varepsilon_{d d}^{0}=0.8$ and suddenly switch to $\varepsilon_{d d}^{f}>1$.

\section{Cigar trap}

For a cigar trap $\gamma=0.1$ the initial state lies in the energy landscape of Fig. 2(a). For $t>0$, we switch to $\varepsilon_{d d}^{f}=1.4$ and an energy landscape of Fig. 2(b). This system is unstable and the BEC undergoes a trajectory (green/grey line) towards the collapse $\left(R_{x}=0\right)$ region. According to the TF scaling parameters $\lambda_{x}(t)$ and $\lambda_{z}(t)$ [solid black lines in Figs. 2(c) and 2(d)], 

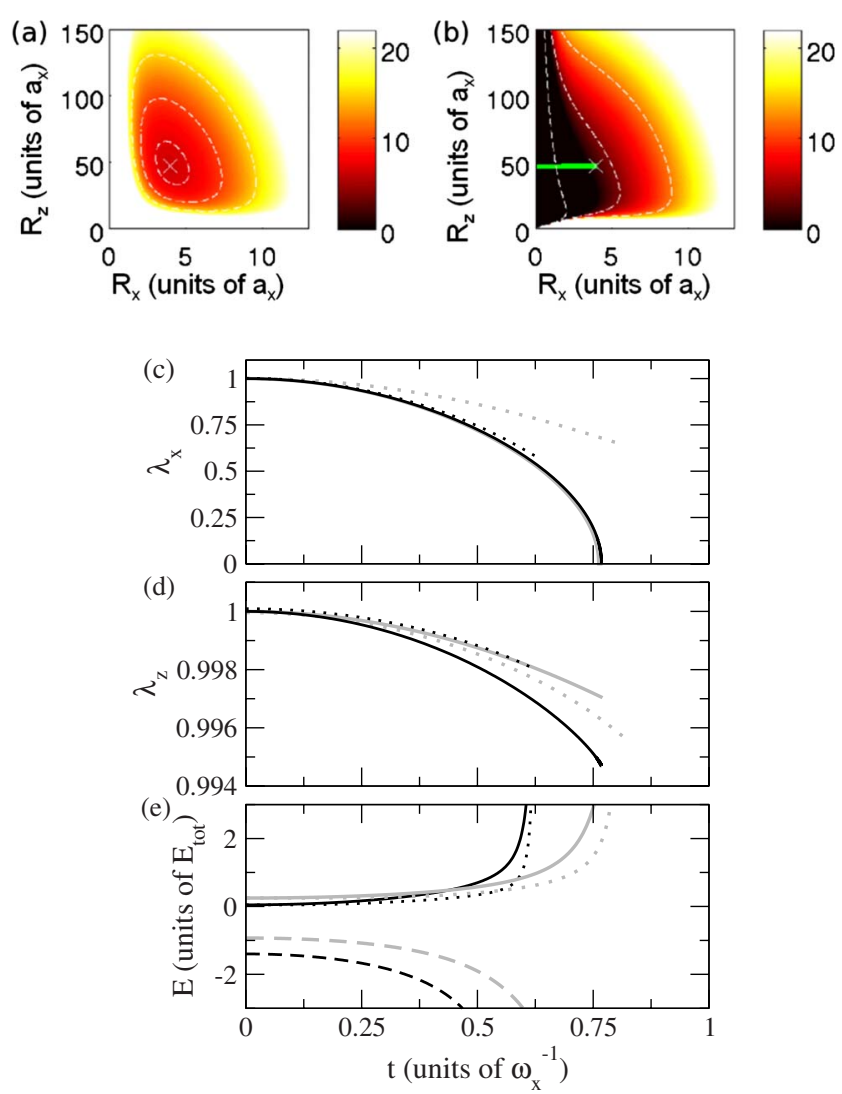

FIG. 2. (Color online) Collapse dynamics in a cigar trap $\gamma$ $=0.1$ for $\varepsilon_{d d}^{0}=0.8$ and $\varepsilon_{d d}^{f}=1.4$. (a) Energy landscape of Eq. (14) at $t=0$. (b) Energy landscape for $t>0$, with the ensuing TF trajectory indicated (green or gray line). Energy is scaled in units of $\hbar \omega_{x}$. (c) $\lambda_{x}(t)$ and (d) $\lambda_{z}(t)$ from the full TF equations of motion (black solid line), the simplified case of Eqs. (23) and (24) (solid gray line), and GPE simulations for $k_{d d}=80$ (grey dotted line) and 2000 (black dotted line). (e) Evolution of the energy components during GPE simulations with $k_{d d}=80$ (grey lines) and $k_{d d}=2000$ (black lines). Shown are the kinetic $E_{k}$ (solid line), zero-point $E_{\text {zp }}$ (dotted line), and dipolar $E_{d}$ (dashed line) energies, renormalized by the total energy $E_{\text {tot }}$.

the condensate accelerates to zero width in the $x$ direction after $t \approx 0.75 \omega_{x}^{-1}$, during which the $z$ width reduces by a very small amount, less than $1 \%$. The collapse is therefore highly anisotropic. Note that this collapse occurs relatively fast since the condensate is initially in an elongated state, close to the collapse threshold. As we will see the time for collapse is strongly dependent on the initial shape of the condensate and therefore on the trap ratio, with more pancake condensates taking longer to collapse.

In our example the BEC is highly elongated both initially and throughout its dynamics. Assuming $\kappa(t) \ll 1$ analytic results for the TF equations of motion can then be obtained. Expanding $f(\kappa)$ as

$$
\frac{f(\kappa)}{1-\kappa^{2}}=1+4 \kappa^{2}+3 \kappa^{2} \ln (\kappa / 2)+O\left(\kappa^{4}\right),
$$

then Eqs. (19) and (20) become, to lowest order,

$$
\begin{gathered}
\ddot{\lambda}_{x} \approx-\omega_{x}^{2} \lambda_{x}+\left[1-\varepsilon_{d d}(t)\right] \frac{\eta \kappa_{0} g(t)}{\lambda_{x}^{3} \lambda_{z}}, \\
\ddot{\lambda}_{z} \approx-\omega_{x}^{2} \gamma^{2} \lambda_{z}+\left[1-\varepsilon_{d d}(t)\right] \frac{\eta \kappa_{0}^{3} g(t)}{\lambda_{x}^{2} \lambda_{z}^{2}} .
\end{gathered}
$$

Recall that $\eta=15 N / 4 \pi m R_{x 0}^{5}$. Analogous equations have been derived for an expanding repulsive $s$-wave BEC [40]. For our time-dependent protocol (21) and to lowest order in $\kappa_{0}$, Eq. (24) has the solution

$$
\lambda_{z}(t)=\cos \left(\omega_{x} \gamma t\right) .
$$

This corresponds to the limit of the noninteracting gas, and the next correction is of order $\kappa_{0}^{2}$. To zeroth order in $\gamma$, $\lambda_{z}(t)=1$, and Eq. (23) has the solution

$$
\lambda_{x}(t)=\frac{1}{\sqrt{2}} \sqrt{(1+\sigma) \cos \left(2 \omega_{x} t\right)+1-\sigma},
$$

where

$$
\sigma=\frac{\eta g_{0} \kappa_{0} \varepsilon_{d d}^{0}}{\omega_{x}^{2}}\left(1-\frac{1}{\varepsilon_{d d}^{f}}\right) .
$$

These simplified analytic solutions can give a remarkably good description of the dynamics. For example, in Fig. 2(c), their prediction of $\lambda_{x}(t)$ is in excellent agreement with the full TF equations of motion. For $\lambda_{z}(t)$ [Fig. 2(d)] deviations are clearly visible, although the dynamics are very slow in this direction.

We have performed GPE simulations of the collapse for a BEC with $k_{d d}=80$. These results [gray dotted lines in Fig. 2(c) and 2(d)] are not in good agreement with the TF predictions. This is because the TF condition for an elongated dipolar BEC (8) is not remotely satisfied [36]. While the BEC approximates a TF profile in the $z$ direction the transverse profile is more akin to a noninteracting Gaussian ground state. Under a much larger interaction strength of $k_{d d}=2000$ (black dotted line), which does satisfy the TF criterion (8), we find good agreement with the TF predictions up until $t$ $\approx 0.65 \omega_{x}^{-1}$. We have evaluated the energy contributions to the GPE as outlined in Appendix B and plotted them in Fig. 2 (e) for both $k_{d d}=80$ (gray lines) and 2000 (black lines). The validity of the TF approximation for $k_{d d}=2000$ is confirmed by the smallness of the zero-point energy in comparison to the other energy contributions. Indeed, it remains small up until $t \approx 0.7 \omega_{x}$, thereby validating the use of the TF approach. At $t=0$ the dipolar energy $E_{d}$ is negative, indicating the attractive configuration of dipoles in the initial state. The dipolar energy remains negative and grows in magnitude as collapse proceeds. Meanwhile the total kinetic energy grows and diverges at $t \approx 0.7 \omega_{x}^{-1}$. The point at which the energies diverge effectively marks the breakdown of the validity of the numerical simulations and the TF approach.

In Fig. 3 we present snapshots from the GPE simulations with $k_{d d}=2000$. The initial density [Fig. 3(i)] is highly elongated along $z$ and approximates the TF inverted parabola. Up to $t \approx 0.4 \omega_{x}^{-1}$ the BEC collapses anisotropically while maintaining the inverted parabola shape. However, from this point in time [Fig. 3(ii)] a local density structure emerges (high- 

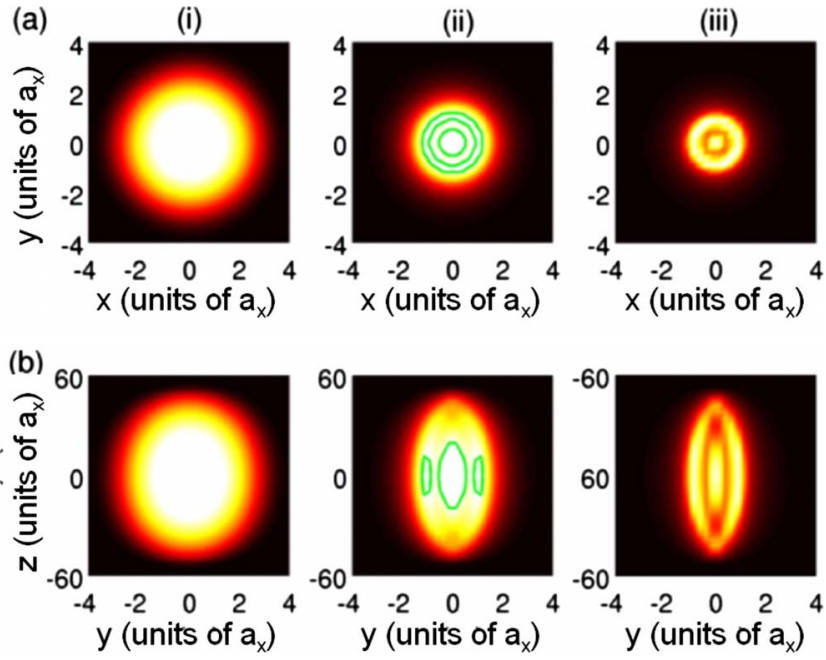

FIG. 3. (Color online) Snapshots of the collapse of the cigar BEC with $k_{d d}=2000$ in the (a) $x-y$ plane and (b) $y-z$ plane at (i) $t$ $=0$, (ii) $t=0.47$, and (iii) $t=0.57 \omega_{x}^{-1}$.

lighted by contours), characterized by modulations in the density. This structure evolves to form a striking arrangement of ellipsoidal shells of high and low density [Fig. 3(iii)], with a high density region in the center of the condensate. The regions of high density grow in population and peak density, and thereby undergo collapse. Note that the development of the density wave structure marks a clear deviation of the system from the parabolic TF solutions.

According to the Bogoliubov spectrum of a homogeneous system (to be discussed further in Sec. IV) the dipolar BEC can be dynamically unstable to phonon modes $[13,35]$. This instability has a strong dependence on angle relative to the polarization direction, and physically this represents the tendency for the system to preferentially align the dipoles in an end-to-end configuration in the polarization direction. This effect gives rise to the highly elongated shell structures observed here.

\section{Pancake trap}

We now consider a BEC within a pancake trap $\gamma=2$. The initial state with $\varepsilon_{d d}^{0}=0.8$ resides in the energy landscape of Fig. 4(a). Following the sudden switch to $\varepsilon_{d d}^{f}=1.4$ and an energy landscape of Fig. 4(b), the BEC follows a trajectory [green (gray) line] towards the collapse $R_{x}=0$ region. According to the TF equations of motion [solid lines in Figs. 4(c) and 4(d)], the condensate accelerates to zero width in the $x$ direction after $t \approx 2 \omega_{x}^{-1}$, while the $z$ width oscillates by approximately $10 \%$. Again, the collapse is highly anisotropic. It is considerably slower than in the cigar trap because the initial condensate is in a more stable state, dominated by repulsive interactions. Note that if the condensate begins in a highly flattened state $\kappa \gg 1$, it elongates over time and its aspect ratio will be reversed. As such no expansion of $\kappa$, analogous to Eqs. (23) and (24), is appropriate.

For $k_{d d}=80$ the TF pancake criterion (9) is satisfied. The corresponding GPE predictions [dotted lines in Figs. 4(c) and $4(d)]$ agree well with the TF predictions up to $t \approx 2 \omega_{x}^{-1}$, with
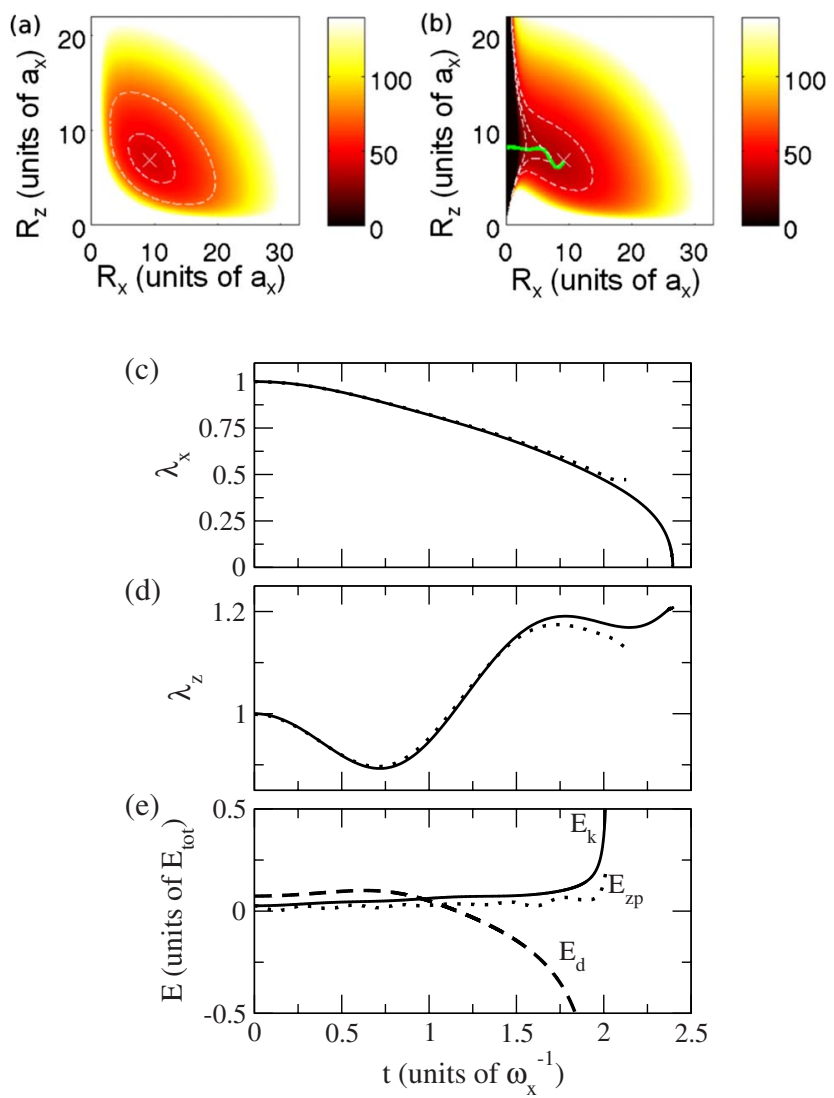

FIG. 4. (Color online) Collapse dynamics in a pancake trap $\gamma$ $=2$ for $\varepsilon_{d d}^{0}=0.8$ and $\varepsilon_{d d}^{f}=1.4$. (a) Energy landscape of Eq. (14) at $t=0$. (b) Energy landscape for $t>0$, with the TF trajectory indicated [green (gray) line]. Energy is scaled in units of $\hbar \omega_{x}$. (c) $\lambda_{x}(t)$ from the TF equations of motion (solid line) and the GPE with $k_{d d}=80$ (dotted line). (d) Same for $\lambda_{z}(t)$. (e) Kinetic $E_{k}$, zero-point $E_{\text {zp }}$ and dipolar $E_{d}$ energies from the GPE, renormalized by the total energy $E_{\text {tot}}$.

the zero-point energy remaining small up until this point. Initially, the dipolar energy is positive due to the dominance of repulsive dipoles in the initial flattened BEC but as the BEC collapses, it becomes negative and diverges. Again, collapse causes a divergence in the kinetic energy.

During the collapse we again see the formation of a local density structure, as shown in Figs. 5(a) and 5(b). These structures are not closed ellipsoidal shells but now rectilinear shells aligned in the $z$ direction. In this case it is likely that the large trap frequency in $z$, which leads to a large excitation energy for axial excitations, suppresses the curvature of the shells. Here the structure features a node of low density for $r=0$ and a single high density shell at finite radius. This structure evolves rapidly with atoms moving away from the center of the condensate to populate the outer shell. This causes the observed flattening in $\lambda_{x}$ at $t \approx 2 \omega_{x}^{-1}$. By later times [Fig. 5(iii)] the shell has elongated axially and shrunk radially, and the system continues to undergo local collapse.

To probe the effect of the trap geometry we also present the collapse dynamics for $\gamma=5$. For $\varepsilon_{d d}^{f}=1.4$ no collapse occurs and so we employ a more extreme value of $\varepsilon_{d d}^{f}=4$. The TF equations of motion [solid lines in Figs. 6(a) and 6(b)] predict that the condensate undergoes shape oscillations 
(a)
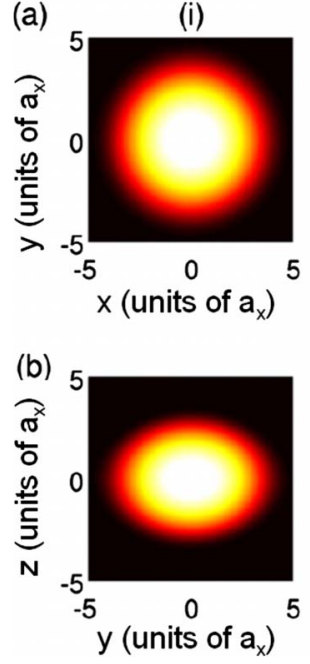

(ii)
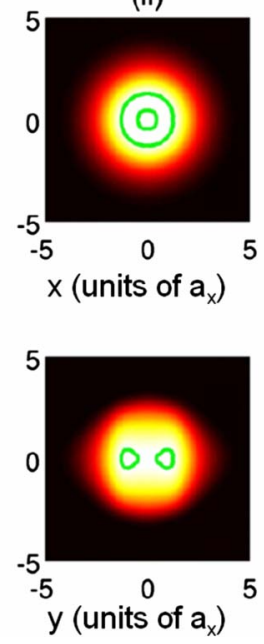

(iii)
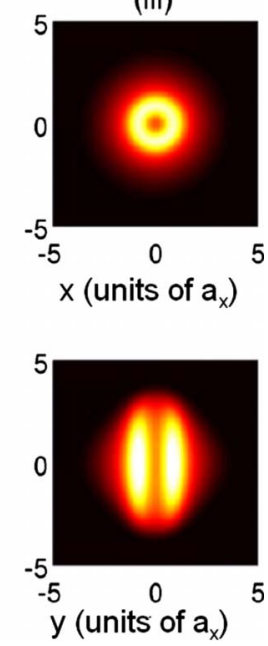

FIG. 5. (Color online) Snapshots of the BEC density during the collapse of Fig. 4 in (a) $x-y$ plane and (b) $y-z$ plane at (i) $t=0$, (ii) $t=1 \omega_{x}^{-1}$, and (iii) $t=1.5 \omega_{x}^{-1}$. Light (dark) regions correspond to high (low) density, while contours in (ii) help to visualize the density structures.

rather than collapse. The GPE results (dotted lines) agree up to $t=1.5 \omega_{x}^{-1}$. During this time the condensate density [Figs. 6(d) and 6(e)(i)] remains approximately an inverted parabola, although weak local density perturbations begin to emerge [Fig. 6(d) and 6(e)(ii)]. However, for $t>1.5 \omega_{x}^{-1}$, the kinetic energy $E_{k}$ diverges to $+\infty$ and $E_{d}$ diverges to $-\infty$. We again observe the development (and collapse) of local density structure [Figs. 6(d) and 6(e)(iii)]. This structure features considerably more shells than our previous example, primarily due to the larger radial extent of the condensate. It also features a central density antinode, rather than a node.

\section{B. Nonadiabatic collapse for $\varepsilon_{d d}^{f}<0$}

Here we start with a stable condensate with $\varepsilon_{d d}^{0}=-0.2$ and suddenly switch to $\varepsilon_{d d}^{f}<-0.5$.

\section{Cigar trap}

In an elongated trap $\gamma=0.2$ we have observed collapse for $\varepsilon_{d d}^{f}=-4$ with the dynamics presented in Fig. 7. Under the TF equations of motion (solid lines), the condensate undergoes large oscillations in the $x$ direction and collapses slowly in $z$. The collapse occurs predominantly in the $z$ direction towards an infinitely thin plane of side-by-side dipoles. This is the opposite to the regime of $\varepsilon_{d d}>0$. Note that the collapse is slow because the condensate is initially in an elongated state where the attractive interactions that induce collapse are weak.

With $k_{d d}=80$ the GPE initial state is in the TF pancake regime (8). The GPE results agree well up to their point of validity at $t \approx 5 \omega_{x}^{-1}$. During this time, the zero-point kinetic energy remains relatively small, and the kinetic and dipolar energies undergo large oscillations due to the radial shape oscillations. At $t \approx 5 \omega_{x}^{-1}, E_{k}$ and $E_{d}$ diverges signifying the limit of validity of the simulations.

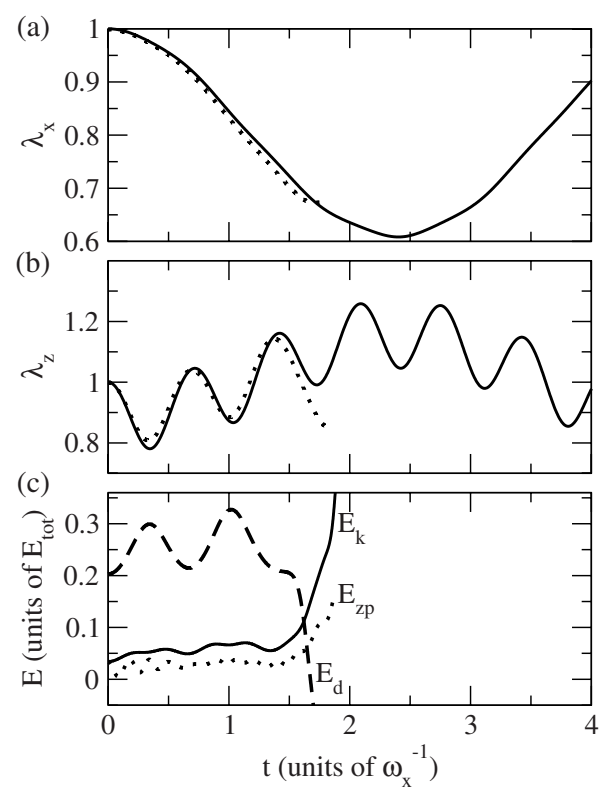

(d)

(i)

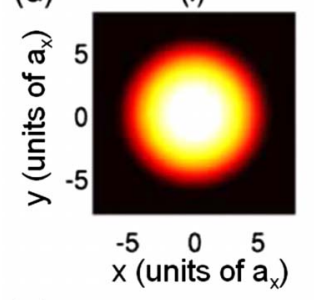

(e)

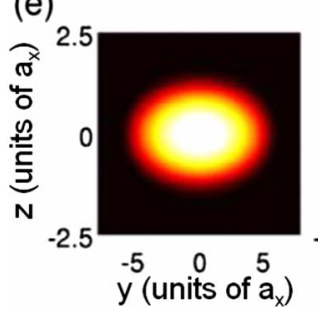

(ii)

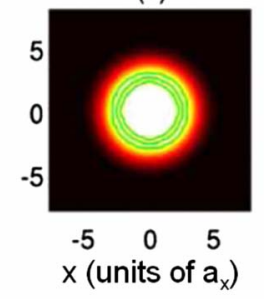

(iii)

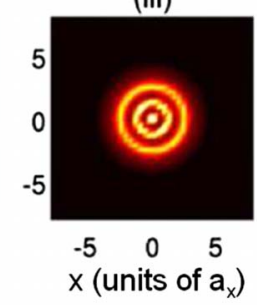

FIG. 6. (Color online) Dynamics in a pancake trap $\gamma=5$ under a sudden change from $\varepsilon_{d d}=0.8$ to 4 . (a) $\lambda_{x}(t)$ and (b) $\lambda_{z}(t)$ from the TF equations (solid line) and the GPE with $k_{d d}=80$ (dotted line). (c) Kinetic $E_{k}$, zero-point $E_{\mathrm{zp}}$, and dipolar $E_{d}$, energies from the GPE simulations. (d)-(e) Density snapshots during the GPE simulations in the (d) $x-y$ plane and (e) $y-z$ plane at (i) $t=0$, (ii) 1 , and (iii) $1.5 \omega_{x}^{-1}$.

Consideration of the condensate density profile during collapse reveals that the condensate develops weak planar density corrugations by $t \approx 4 \omega_{x}^{-1}$ [Figs. 7(d) and 7(e)(ii)]. These become amplified into a striking planar density perturbation of approximately ten localized planes of dipoles, aligned in the $x-y$ plane [Figs. 7(d) and 7(e)(iii)]. This is the same phenomenon as observed earlier but with perpendicular orientation due to the fact that the $\varepsilon_{d d}<0$ dipoles are now attractive when side-by-side.

\section{Pancake trap}

For $\varepsilon_{d d}^{f}=-0.8$, the condensate dynamics within a pancake trap $\gamma=2$ are presented in Fig. 8. According to the TF equa- 


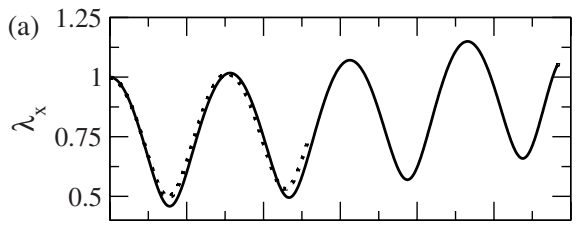

(b)
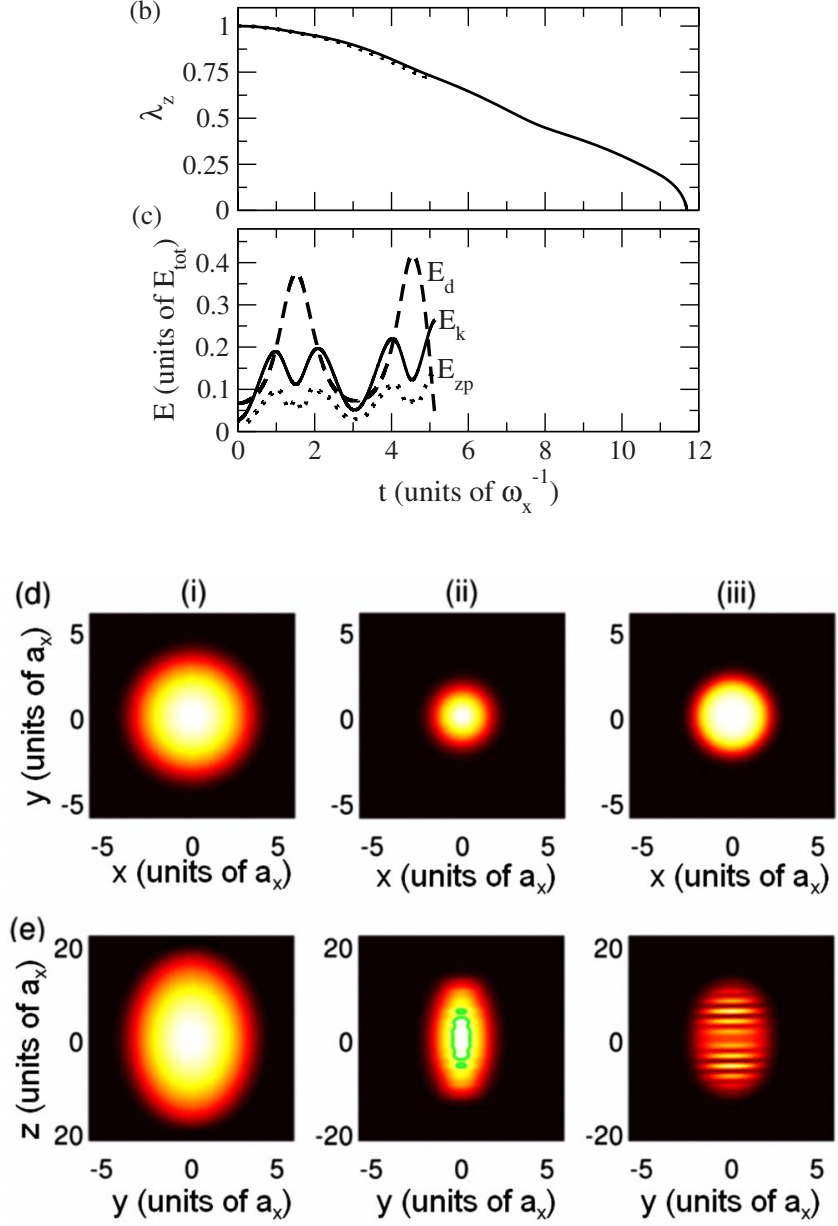

FIG. 7. (Color online) Collapse dynamics in a cigar trap $\gamma$ $=0.2$ for $\varepsilon_{d d}^{0}=-0.2$ and $\varepsilon_{d d}^{f}=-4$. (a) $\lambda_{x}(t)$ and (b) $\lambda_{z}(t)$ from the TF equations of motion (solid line) and the GPE with $k_{d d}=80$ (dotted line). (c) Kinetic $E_{k}$, zero-point $E_{\text {zp }}$, and dipolar $E_{d}$ energies. (d), (e) GPE density in the (d) $x-y$ plane and (e) $y-z$ plane at (i) $t=0$, (ii) 4.4 , and (iii) $5 \omega_{x}^{-1}$.

tions of motion, $\lambda_{z}(t)$ accelerates to zero within $t \approx 0.6 \omega_{x}^{-1}$ while $\lambda_{x}(t)$ decreases more slowly. Taking $k_{d d}=80$ the initial ground state satisfies the pancake TF criteria (9) and we see excellent agreement with the TF predictions up to $t$ $\approx 0.6 \omega_{x}^{-1}$, during which $E_{\text {zp }}$ remains relatively small. However, beyond this point $E_{\text {zp }}$ diverges, as does $E_{k}$ and $E_{d}$.

As with the cigar case, the condensate density develops planar density perturbations, as shown in Figs. 8(d) and 8(e)(ii) and (iii). However, given that the pancake system is narrower in $z$, only two planes of high density emerge.

\section{GENERAL PROPERTIES OF COLLAPSE}

We now map out the general behaviour of the important length scales and time scales of the collapse, and the critical trap ratio that can stabilize against collapse.

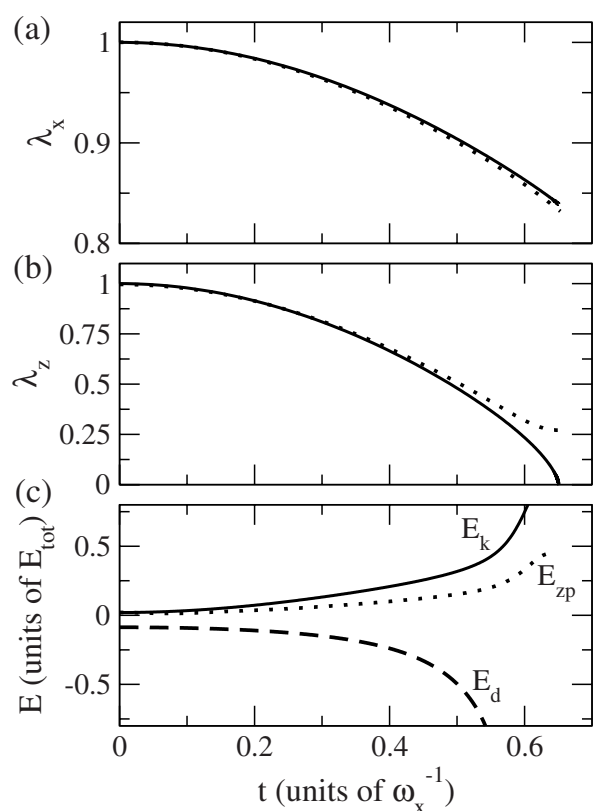

(d)

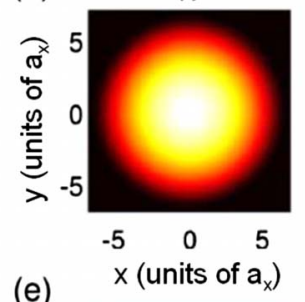

(e)

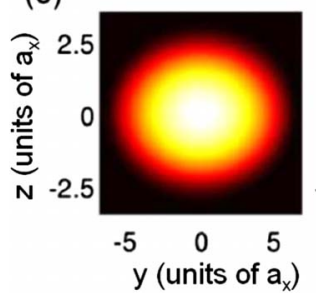

(ii)
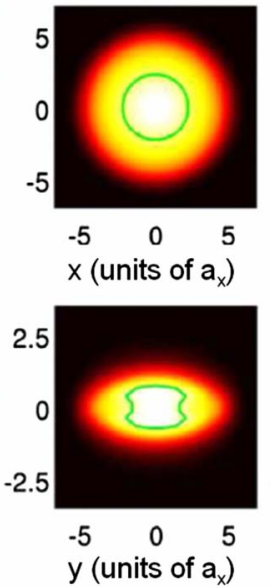

(iii)
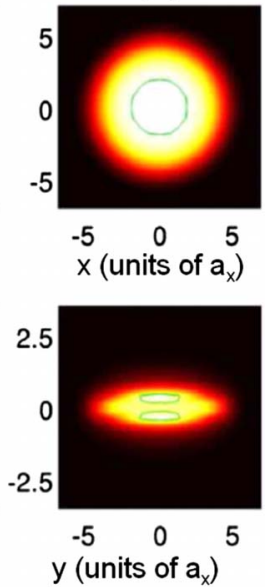

FIG. 8. (Color online) Collapse dynamics in a pancake trap $\gamma$ $=2$ under a sudden change from $\varepsilon_{d d}=-0.2$ to -0.8 . (a) $\lambda_{x}(t)$ and (b) $\lambda_{z}(t)$ according to the full TF equations of motion (solid line) and GPE simulations with $k_{d d}=80$ (dotted line). (c) Kinetic $E_{k}$, zeropoint $E_{\mathrm{zp}}$, and dipolar $E_{d}$ energies from the GPE, renormalized by the total energy $E_{\text {tot }}$ (d), (e) Density snapshots in the (d) $x-y$ and (e) $y-z$ planes at (i) $t=0$, (ii) 0.42 , and (iii) $0.53 \omega_{x}^{-1}$.

\section{A. Length scales for collapse}

A homogenous dipolar BEC is unstable to periodic density perturbations (phonons) when $\varepsilon_{d d}>1$ or $\varepsilon_{d d}<-0.5$ [13]. This can be seen immediately from the Bogoliubov dispersion relation between the energy $E_{B}$ and momentum $p$ for phonons in the gas, given by

$$
E_{B}=\sqrt{\left(\frac{p^{2}}{2 m}\right)^{2}+2 g n\left[1+\varepsilon_{d d}\left(3 \cos ^{2} \theta-1\right)\right] \frac{p^{2}}{2 m}} .
$$

A mode with energy $E_{B}$ evolves as $\exp \left(i E_{B} t / \hbar\right)$ and so when $E_{B}$ becomes imaginary, the mode grows exponentially, i.e., a dynamical instability. This dispersion relation depends on $\theta$, 
(a)

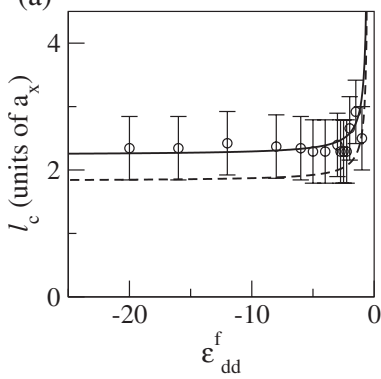

(b)

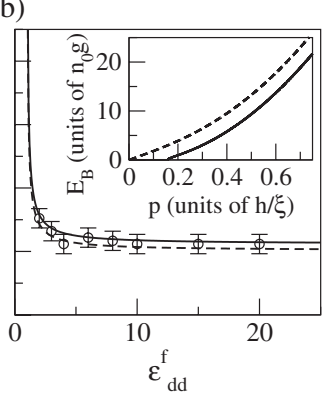

FIG. 9. Length scale of collapse $l_{c}$ as a function of $\varepsilon_{d d}^{f}$ according to GPE simulations (circles) and the predictions of Eqs. (31) and (32) for a homogeneous system $A=B=1$ (dashed lines) and for a trapped system $A \neq B \neq 1$ (solid line). (a) The regime $\varepsilon_{d d}<-0.5$, assuming a cigar-shaped BEC with $\gamma=0.2, \varepsilon_{d d}^{0}=-0.2$, and $k_{d d}=$ -80 . For the cigar prediction (solid line) we assume a TF transverse profile for which $B=\sqrt{3 / 2}$. (b) The regime $\varepsilon_{d d}>1$, assuming a pancake geometry $\gamma=5$ with $\varepsilon_{d d}^{0}=0.8$ and $k_{d d}=80$. For the pancake prediction (solid line) we assume a TF axial profile for which $A$ $=\sqrt{5 / 4}$. Inset: Homogeneous Bogoluibov spectrum of Eq. (28) for $\varepsilon_{d d}=1.4$ and for $\theta=0$ (dotted line) and $\theta=\pi / 2$ (dot-dashed line). Note that the error bars in the GPE results arise from the grid discretization.

the angle between the momentum of the phonon and the external polarizing field. For $\varepsilon_{d d}>1$, phonons propagating perpendicularly to the polarization direction $(\theta=\pi / 2)$ undergo a dynamical instability while no instability occurs along the polarization direction $(\theta=0)$. This is illustrated in Fig. 9(b) (inset) which plots the spectrum (28) for $\varepsilon_{d d}=1.4$ and for the polarizations $\theta=0$ (dashed line) and $\theta=\pi / 2$ (solid line); the point where the solid line touches zero marks the transition to instability. In an infinite and initially homogeneous system we expect this instability to break the condensate up into a lattice of filaments, i.e., cylindrical structures aligned along the polarization direction. In trapped condensates, shells or planes (aligned along $z$ ) may be favored instead. Meanwhile, when $\varepsilon_{d d}<-0.5$ (we remind the reader that we mean in this case that $C_{d d}<0$ ), it is phonons propagating along the polarization direction $(\theta=0)$ that undergo a dynamical instability while no instability occurs in the perpendicular direction $(\theta=\pi / 2)$. This suggests an instability towards stratification into planes lying perpendicular to the polarization axis. These predictions are consistent with our observations in the previous section.

We can estimate the characteristic length scale of the collapse structures from the homogeneous Bogoliubov spectrum (28). If $p_{c}$ is the critical momentum for which the dispersion relation passes through zero energy (which signifies the onset of dynamical instability), then we can expect the characteristic length scale to be $l_{c}=2 \pi \hbar / p_{c}$. Although we are concerned with trapped, inhomogeneous condensates, the same length scale $l_{c}$ will apply providing the system is of size $R$ $\gg l_{c}$. From Eq. (28) it is straight forward to show that this length scale is given by

$$
l_{c}=A \frac{\sqrt{2} \pi \xi}{\sqrt{\varepsilon_{d d}-1}} \text { for } \varepsilon_{d d}>1,
$$

$$
l_{c}=B \frac{\sqrt{2} \pi \xi}{\sqrt{\left|1+2 \varepsilon_{d d}\right|}} \text { for } \varepsilon_{d d}<-0.5 .
$$

Here $\xi=1 / \sqrt{8 \pi n_{0}\left|a_{\mathrm{s}}\right|}$ is the $s$-wave healing length at the condensate center. $A$ and $B$ are factors that take account of the trapping and are unity in a homogenous system.

We can improve the applicability of our length scale predictions to inhomogeneous systems by taking into account the trapping in one or two directions. In an infinite cylindrical BEC with tight radial trapping, such that the radial density profile is a Gaussian, it is known that the axial speed of sound is reduced by a factor of $\sqrt{2}$ in comparison to a uniform system with the same peak density $n_{0}$ [43]. This rescaling of the dispersion relation arises because the average density is in fact given by $n_{0} / 2$. In general, we can obtain the average density for both cigar and pancake condensates by integrating out the tightly confined direction. We will perform this for the relevant cases of Gaussian and parabolic (TF) density profiles. The resulting modification to the dispersion relations along the weakly confined direction(s) gives the trapping parameters $A$ and $B$ which occur in Eqs. (29) and (30). For $\varepsilon_{d d}>1$ the interesting case is that of a pancake, for which $A \rightarrow A_{\text {Gauss }}=2^{1 / 4} \approx 1.2$ in the Gaussian case and $A \rightarrow A_{\mathrm{TF}}=\sqrt{5 / 4} \approx 1.1$ in the TF case. For $\varepsilon_{d d}$ $<-0.5$ the interesting case is that of a cigar, for which $B$ $\rightarrow B_{\text {Gauss }}=\sqrt{2} \approx 1.4$ in the Gaussian case and $B \rightarrow B_{\mathrm{TF}}=\sqrt{3 / 2}$ $\approx 1.2$ in the $\mathrm{TF}$ case.

In order to relate our numerical results on adiabatic and nonadiabatic collapse to the unstable modes of Eq. (28), let us consider the case of positive $\varepsilon_{d d}$. For $\varepsilon_{d d}=1$ the collapse length scale is infinite. If $\varepsilon_{d d}$ is increased adiabatically then $l_{c}$ decreases. At some critical $\varepsilon_{d d}, l_{c}$ becomes equal to the condensate size $R$ and a dynamical instability occurs on the length scale of $R$. Thus, according to Eq. (28), if the point of collapse is reached adiabatically, global collapse will occur. This picture is qualitatively consistent with our observations in Sec. II in the range $-1 \leq \varepsilon_{d d} \leqslant 2$, where we observed that adiabatic collapse proceeds in a global manner. However, outside of this regime we saw evidence for adiabatic local collapse, which we attributed to the presence of a roton minimum in the excitation spectrum. The homogeneous dispersion relation (28) does not have a roton minimum and in order to introduce one it is necessary to explicitly include a trap in at least one dimension. In highly cigar-shaped or pancake-shaped systems the roton minimum occurs in the dispersion relation for low-energy excitations along the weakly confined direction. The momentum at which the roton minimum occurs $p_{r}$, defines a length scale $l_{r}=2 \pi \hbar / p_{r}$ which is usually closely related the system size in the tightly trapped direction $[23,24]$. Adiabatic local collapse is therefore expected to take place on length scales $l_{r}$ where the roton minimum touches the zero energy axis. We have compared simple analytic predictions for the dispersion relations of an infinite cigar [22] and an infinite pancake [23], which do include a roton minimum, with the homogeneous result (28). We find that the value of $l_{c}$ can significantly differ from the predictions of Eqs. (29) and (30) when the system is close to the collapse threshold, but quickly become very similar as we move deeper into the collapse regime. Thus, 
we expect that an adiabatic collapse experiment could provide clear evidence for the presence of a roton minimum in the excitation spectrum, but a nonadiabatic collapse experiment would be less conclusive. To tackle this problem properly one should numerically obtain the full Bogoliubov spectrum of a trapped system [37].

We now consider the situation where we suddenly switch from $\varepsilon_{d d}^{0}<1$ to $\varepsilon_{d d}^{f} \gg 1$. Here we go from the regime where $l_{c} \gg R$ to $l_{c}<R$, and a dynamical instability occurs on a local scale. Although the dynamical instability evolves for all length scales in the range $l>l_{c}$, the imaginary energy eigenvalue is largest for $l=l_{c}$ and this unstable mode dominates the system. This is consistent with our observations in Sec. III where, following a sudden change in $\varepsilon_{d d}$, collapse evolves mainly through local density structures.

We now specifically consider the case where collapse is induced suddenly by modifying the $s$-wave interactions, while the dipolar interactions remains constant. Then we can rewrite Eqs. (29) and (30) as

$$
\begin{gathered}
l_{c}=A \sqrt{\frac{\pi \varepsilon_{d d}^{f}}{4 n_{0} a_{d d}\left(\varepsilon_{d d}^{f}-1\right)}} \text { for } \varepsilon_{d d}>1, \\
l_{c}=B \sqrt{\frac{\pi \varepsilon_{d d}^{f}}{4 n_{0}\left|a_{d d}\left(1+2 \varepsilon_{d d}^{f}\right)\right|}} \text { for } \varepsilon_{d d}<-0.5 .
\end{gathered}
$$

These predictions are plotted in Figs. 9(a) and 9(b), for the regimes of $\varepsilon_{d d}<-0.5$ and $\varepsilon_{d d}>1$, respectively. We have conducted a series of GPE simulations for trapped dipolar BECs to determine the true collapse length scale (defined as the distance between peaks in adjacent shells), with these predictions being shown by the circles. Note that in Fig. 9(a) we employ a cigar-shaped BEC with $\gamma=0.2$ and $\varepsilon_{d d}^{0}=-0.2$, and in Fig. 9(b) we employ a pancake-shaped BEC with $\gamma$ $=5$ and $\varepsilon_{d d}^{0}=0.8$. Even in the homogeneous limit $A=B=1$ (dashed lines) the analytic predictions for $l_{c}$ are in very good agreement with the simulations. Examination of the GPE solutions reveals that the density profile in the tightly confined direction is closely approximated by the TF profile. The analytic results using the appropriate trapping parameters $(A$ $=\sqrt{5 / 4}$ and $B=\sqrt{3 / 2}$ ) are shown by solid lines in Fig. 9. With these trapping parameters included the agreement becomes excellent, and clearly demonstrates the importance of taking the trapping into account.

\section{B. Time for global collapse}

We now make some simple predictions for the characteristic collapse time $\tau_{c}$. Having demonstrated that the TF model gives a good description of collapse we will employ it exclusively here. Since the TF equations of motion cannot describe local collapse, our analysis is limited to global collapse. Furthermore we will only consider nonadiabatic collapse since the time scale for adiabatic collapse is technically infinite.

Experimentally, the collapse time is the time at which a "sudden" depletion of the condensate occurs due to threebody loss [9]. The suddenness arises because of the dramatic scaling of losses with time: the rate of three-body loss scales
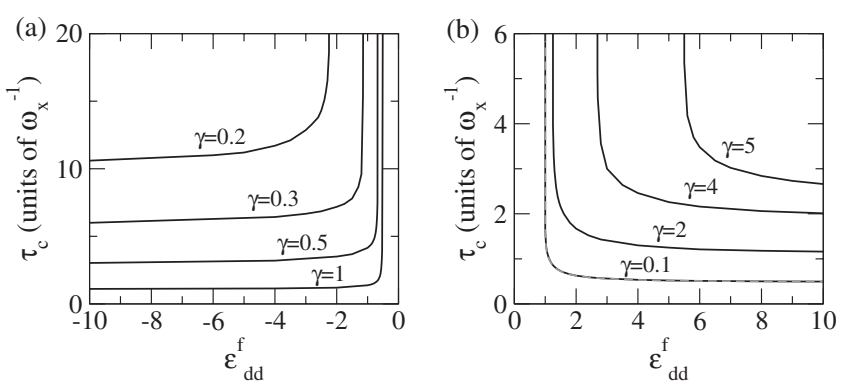

FIG. 10. Collapse time $\tau_{c}$ following a sudden change in $\varepsilon_{d d}$ according to the TF equations of motion. Various trap ratios are presented. (a) $\varepsilon_{d d}^{0}=-0.2$ and $\varepsilon_{d d}^{f}<0$. (b) $\varepsilon_{d d}^{0}=0.8$ and $\varepsilon_{d d}^{f}>0$. For the case of $\gamma=0.1$ we also plot the analytic expression of Eq. (33) (grey dashed line).

as $n^{3}$, and the density $n$ itself accelerates in amplitude during collapse. Consequently, a good estimate for $\tau_{c}$ is the time over which the peak density diverges or, equivalently, the time over which one or more radii tend to zero [18].

The TF collapse time can be obtained, in general, by numerical solution of the TF equations of motion. However, for highly elongated BECs, Eqs. (25) leads to an analytic form for $\tau_{c}$ given by

$$
\tau_{c}=\frac{1}{2} \arccos \left[\frac{\sigma-1}{\sigma+1}\right],
$$

where $\sigma$ is given by Eq. (27). It should be noted that in the limit $\varepsilon_{d d}^{f} \rightarrow \infty$, Eq. (27) becomes

$$
\lim _{\varepsilon_{d d}^{f} \rightarrow \infty} \sigma=\varepsilon_{d d}^{0} \frac{\eta g_{0} \kappa_{0}}{\omega_{x}^{2}},
$$

where we recall that $\eta=15 N / 4 \pi m R_{x 0}^{5}$. Thus the limiting value of $\tau_{c}$ is determined only by the initial parameters $\gamma$ and $\varepsilon_{d d}^{0}$.

In Figs. 10(a) and 10(b) we show how $\tau_{c}$ depends on the final interaction parameter $\varepsilon_{d d}^{f}$ for initial values of $\varepsilon_{d d}^{0}$ $=-0.2$ and 0.8 , respectively. For weak interactions $\tau_{c} \mathrm{di}-$ verges as the interactions become too weak to induce collapse, while in the limit of large interactions (positive or negative $\varepsilon_{d d}$ ), $\tau_{c}$ tends towards a finite value, as expected. For $\varepsilon_{d d}<0$, elongated systems become more stable and are the slowest to collapse. In contrast, for $\varepsilon_{d d}>0$, elongated systems are least stable and therefore the fastest to collapse. Note that for the highly elongated case of $\gamma=0.1$, the analytic collapse time of Eq. (33) is in excellent agreement with the full TF equations of motion.

\section{Critical trap ratio for global collapse}

In the range of $\varepsilon_{d d}$ considered in this work, there exists a critical trap ratio $\gamma_{c}$ which can stabilize the parabolic $\mathrm{TF}$ solutions against global collapse. As we showed in Sec. III, if collapse is approached adiabatically this critical trap ratio is fixed, being $\gamma_{c}=5.2$ for positive $\varepsilon_{d d}$ and $\gamma_{c}=0.19$ for negative $\varepsilon_{d d}$. However, if collapse is induced nonadiabatically, the critical trap ratio becomes a function of $\varepsilon_{d d}^{0}$. To examine this threshold for global collapse in more detail, Fig. 11 plots 

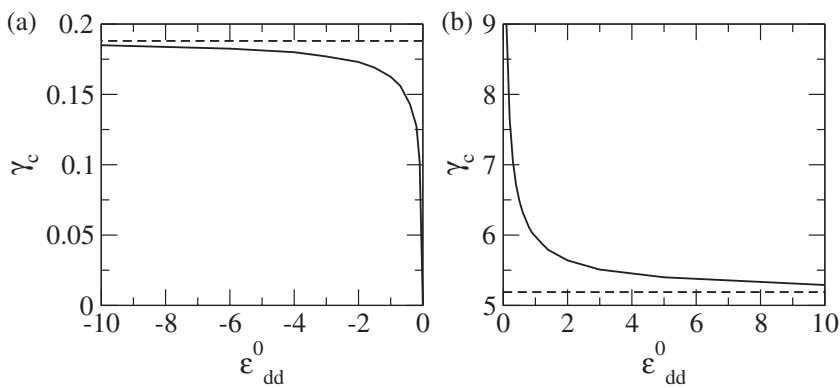

FIG. 11. Critical trap ratio $\gamma_{c}$ that stabilizes the BEC against collapse as a function of initial interaction strength $\varepsilon_{d d}^{0}$, according to the full TF equations of motion. The final interaction strength $\varepsilon_{d d}^{f}$ $=10^{4}$ is so large that it is effectively the infinite limit.

the critical trap ratio $\gamma_{c}$ as a function of the initial interaction parameter $\varepsilon_{d d}^{0}$ for both negative and positive $\varepsilon_{d d}$. Note that we have considered $\left|\varepsilon_{d d}^{f}\right|=10^{4}$, which is so large that it effectively behaves as the infinite limit. For $\varepsilon_{d d}^{0}<0, \gamma_{c}$ tends towards zero for $\varepsilon_{d d}^{0} \rightarrow 0$, and in the opposing limit of $\varepsilon_{d d}^{0}$ $\rightarrow-\infty$ it increases asymptotically towards the adiabatic value $\gamma_{c}=0.19$. Conversely, for $\varepsilon_{d d}^{0}>0, \gamma_{c}$ diverges as $\varepsilon_{d d}^{0} \rightarrow 0$, and in the opposing limit of $\varepsilon_{d d} \rightarrow \infty$ it decreases towards the static value $\gamma_{c}=5.17$. Note that, providing $\left|\varepsilon_{d d}^{0}\right| \gg 0, \gamma_{c}$ varies only weakly with $\varepsilon_{d d}^{0}$ and becomes very close to the static value of $\gamma_{c}$.

\section{COMPARISON WITH EXPERIMENT}

In a recent series of experiments the Stuttgart group demonstrated the collapse of a dipolar condensate $[8,9]$. A Feshbach resonance was employed to give time-dependent control over $a_{s}$ and therefore $\varepsilon_{d d}$. In Ref. [8], $a_{s}$ was reduced slowly (over several trap periods) to probe the stability of the ground state to collapse. For $\gamma=1$, collapse was observed for $\varepsilon_{d d} \approx 1$, which is in good agreement with the static solutions in Fig. 1(a). For $\gamma \approx 10$, the BEC was stabilized under purely dipolar interactions, i.e., the limit $\varepsilon_{d d} \rightarrow \infty$, in good qualitative agreement with our understanding of the role of trap geometry.

In Ref. [9], collapse was observed in an almost spherical trap. From an initial BEC at $t=0$ with $a_{s}^{0}=1.59 \mathrm{~nm}$, the scattering length was reduced linearly to a final value $a_{s}^{f}$ $=0.27 \mathrm{~nm}$ over a time scale $\tau \sim 1 \mathrm{~ms}$. The BEC was observed to collapse anisotropically towards a narrow cigar shape, aligned in $z$, over a time scale of $\tau_{c}^{\exp } \approx 1.5 \mathrm{~ms}$. We will see that this time scale is sufficiently long that local collapse is not induced. Following this initial collapse an explosion occurred, resulting in a spectacular state with a shape resembling that of a $d$-wave orbital. Numerical simulations of the GPE with three-body loss were in excellent agreement with the observed dynamics [9].

The experimental collapse appears to occur globally with no signs of local collapse. To resolve the issue of the apparent absence of local collapse, we have simulated the experimental dynamics based on $N=20000$ atoms and a fully anisotropic trap $\left(\omega_{x}, \omega_{y}, \omega_{z}\right)=2 \pi \times(660,400,530) \mathrm{Hz}$. Under linear ramping of $a_{s}$ over time $\tau=1 \mathrm{~ms}$, our results in Fig. 12 (a)
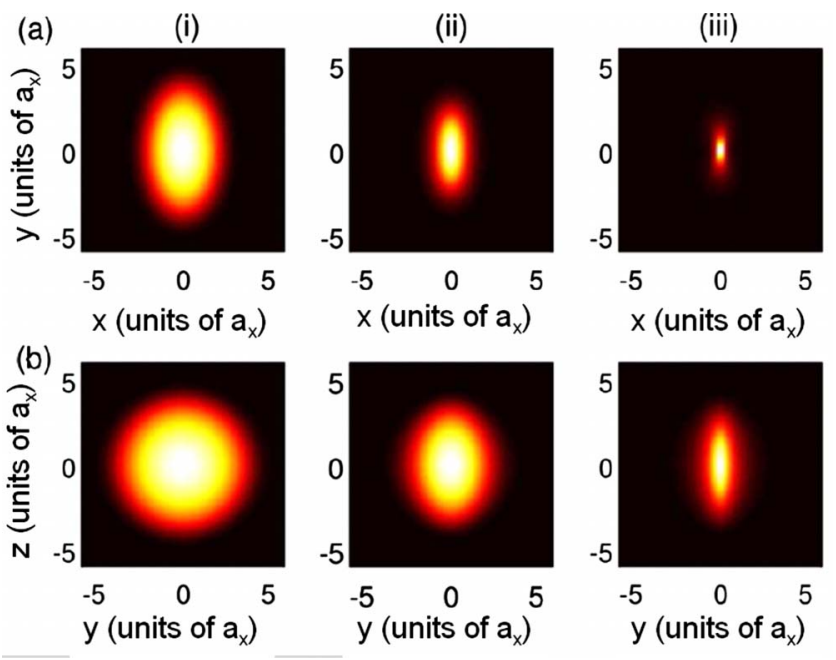

FIG. 12. (Color online) Collapse dynamics in the Stuttgart system under the linear decrease of $a_{s}$ over time $\tau_{r}=1 \mathrm{~ms}$. Density in the (a) $x-y$ and (b) $y-z$ plane at (i) $t=0$, (ii) $t=3.1$, and (iii) $t$ $=3.7 \omega_{x}^{-1}$.

confirm that the condensate collapses globally rather than locally. The condensate collapses, mainly in the $x-y$ plane, and forms a very narrow cigar-shaped BEC. Because $\omega_{x}>\omega_{y}$, the transverse collapse is quickest in the $x$ direction. The elongated collapsed state [Fig. 12(iii)] forms after a collapse time $\tau_{c} \approx 1 \mathrm{~ms}$. This is quicker than the time scales reported in Ref. [9], where it is known that eddy currents suppress the applied change in $a_{s}$ and effectively extend the ramping time by a factor of 2 or 3 . This does not affect our qualitative results but merely slows down the collapse. Indeed, if we employ a ramping time of $\tau=2 \mathrm{~ms}$, we find $\tau_{c}$ $\approx 1.6 \mathrm{~ms}$, in agreement with Ref. [9].

The experimental ramping time is far from being sudden since it is of the order of the trap period that characterizes the internal dynamics of the BEC. We can therefore expect that global collapse will be initiated well before any local instabilities. Indeed, if we make a more sudden change of $a_{s}$, e.g., $\tau=0$, then we see in Fig. 13 that local collapse now occurs. However, within this noncylindrically symmetric geometry we observe the formation of parallel density stripes, rather than shells. Again, the $x$ direction collapses towards zero width. However, the $y$ direction does not shrink globally but develops a corrugated structure that enables the dipoles to predominantly align along $z$. These stripes become amplified and collapse themselves.

\section{CONCLUSIONS}

We have studied the collapse dynamics of a dipolar BoseEinstein condensate, triggered either by an adiabatic or nonadiabatic change in the dipolar-to- $s$-wave ratio $\varepsilon_{d d}=C_{d d} / 3 g$. In general, the collapse occurs anisotropically and is driven by the dipoles seeking to line up end-to-end for $\varepsilon_{d d}>0$ and side-by-side for $\varepsilon_{d d}<0$. In the case of adiabatic collapse, where the ground state solutions are followed up until the instant of collapse, we observe both global and local collapse. In the range $-1 \lesssim \varepsilon_{d d} \lesssim 2$ we find global collapse to- 
(a)
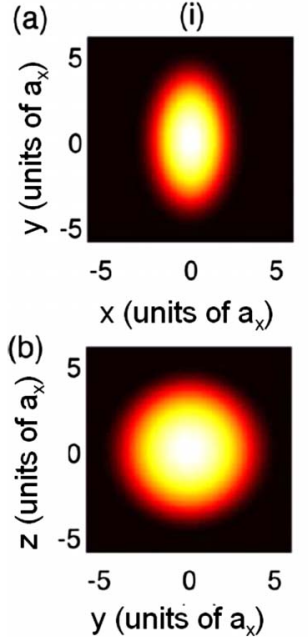

(ii)

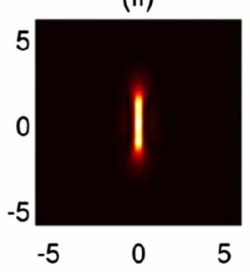
$x$ (units of $a_{x}$ )

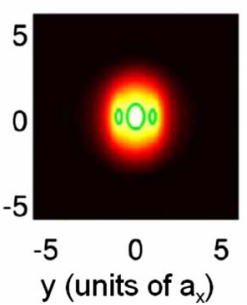

(iii)

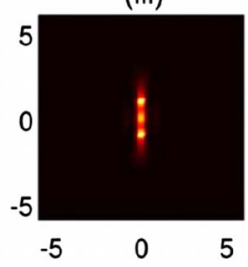
$x$ (units of $\left.a_{x}\right)$

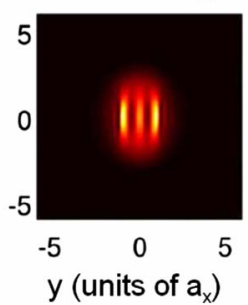

FIG. 13. (Color online) Collapse dynamics in the Stuttgart system under the sudden decrease in $a_{s}$. Density in the (a) $x$ - $y$ plane and (b) $y-z$ plane at (i) $t=0$, (ii) $t=1.08$, and (iii) $t=1.16 \omega_{x}^{-1}$.

wards a single line or plane of dipoles. Outside of this range we have seen adiabatic local collapse which we suggest is a signature of a roton minimum in the excitation spectrum (note that adiabatic local collapse is not possible in $s$-wave condensates). Similar theoretical predictions have also recently been reported by Bohn et al. [19]. Note that care must be taken to distinguish such local collapse from the results of nonadiabatic collapse.

If collapse is triggered nonadiabatically via a sudden change in $\varepsilon_{d d}$, the instability can jump to length scales much less than the condensate size, resulting in local collapse. We have analyzed this instability over the considerable range $-10 \lesssim \varepsilon_{d d} \lesssim 20$. This instability can be understood in terms of the amplification of dynamically unstable phonon modes. For a cylindrically symmetric condensate, the system develops a periodic structure of density shells for $\varepsilon_{d d}>0$ or disks for $\varepsilon_{d d}<0$, which become amplified and subsequently collapse.

We applied the TF equations of motion, previously used to model oscillatory and expansion dynamics, to study nonadiabatic collapse of the condensate. We showed that this method is valid providing that zero-point kinetic effects remain small throughout. This can be ensured by employing a TF condensate initially, and suddenly switching the interactions deep into the collapse regime. The predictions are in excellent agreement with the full Gross-Pitaevskii equation for the majority of the collapse dynamics. The TF predictions fail when the condensate develops local density structures and thereby deviates from the TF parabolic density profile.

Our results are consistent with the experiment by Lahaye et al. [9]. There the increase of $\varepsilon_{d d}$ was sufficiently slow to ensure that global collapse dominates the system. However, by changing the interactions more suddenly, it should be possible to induce local collapse of the condensate. A rich variety of structures can be formed depending upon the orientation of the dipoles and the aspect ratio of the trap. These structures are related to those that have been predicted to occur in the ground state, but during local collapse they

become amplified. Such transient structures could be observed experimentally by Bragg scattering of light.

\section{ACKNOWLEDGMENTS}

This work was funded by the Government of Canada (N.G.P.), NSERC (D.H.J.O.D.), and ARC (C.T., A.M.M.). We thank C. A. Sackett for discussions.

\section{APPENDIX A: GAUSSIAN VARIATIONAL SOLUTIONS}

When the TF approximation is not valid (e.g., for weak interactions), it can be appropriate to employ a Gaussian ansatz. We consider a cylindrically symmetric Gaussian ansatz of the form $[8,14]$

$$
\psi_{g}=\sqrt{\frac{\kappa N}{\pi^{3 / 2} l_{x}^{3}}} \exp \left[-\frac{1}{2 l_{x}^{2}}\left(x^{2}+y^{2}+\kappa^{2} z^{2}\right)\right],
$$

where $l_{x}$ is the transverse width and $\kappa$ is the aspect ratio. The energy of this ansatz is

$$
\frac{E}{N \hbar \omega_{x}}=\frac{1}{2 l_{x}^{2}}+\frac{\kappa^{2}}{4 l_{x}^{2}}+\frac{l_{x}^{2}}{2}+\frac{\gamma^{2} l_{x}^{2}}{2 \kappa^{2}}+\frac{\kappa k_{s}\left[1-\varepsilon_{d d} f(\kappa)\right]}{\sqrt{2 \pi} l_{x}^{3}} .
$$

Variational solutions are then obtained by minimizing this with respect to $l_{x}$ and $\kappa$.

\section{APPENDIX B: NUMERICAL SOLUTION OF THE DIPOLAR GPE}

A split-step fast Fourier transform method is employed to evolve $\psi(\mathbf{r})$ on a three-dimensional spatial grid [37], typically with $64^{3}$ points. The dipolar atomic potential in $k$ space is given by

$$
\widetilde{U}_{d d}(k)=\frac{4 \pi C_{d d}}{3}\left(\frac{3 k_{z}^{2}}{k^{2}}-1\right),
$$

where $k_{x}, k_{y}$, and $k_{z}$ are the Cartesian wave vectors and $k^{2}$ $=k_{x}^{2}+k_{y}^{2}+k_{z}^{2}$. By combining Eq. (5) with the convolution theorem, the dipolar mean-field potential is given by $\Phi_{d d}(\mathbf{r})=\mathcal{F}^{-1}\left[\tilde{U}_{d d}(k) \widetilde{n}(k)\right]$, where the inverse Fourier transform, denoted by $\mathcal{F}^{-1}$, is performed numerically using a standard fast Fourier transform algorithm. For pancake and spherical systems we employ the corrections to $\widetilde{U}_{d d}(k)$ outlined in Ref. [37]. Ground-state solutions of the dipolar GPE are found by propagation in imaginary time $t \rightarrow-i t$. From a suitable initial guess and with renormalization at each time step, the GPE converges to the ground state of the system.

To monitor the size of the BEC we evaluate $\left\langle z^{2}\right\rangle$ and $\left\langle x^{2}\right\rangle$ and relate them to the TF radii $R_{z}$ and $R_{x}$ via

$$
\begin{aligned}
& \left\langle z^{2}\right\rangle=\int d \mathbf{r} z^{2}|\psi|^{2}=\frac{N}{7} R_{z}^{2}, \\
& \left\langle x^{2}\right\rangle=\int d \mathbf{r} x^{2}|\psi|^{2}=\frac{N}{7} R_{x}^{2} .
\end{aligned}
$$


To compare the numerical widths directly with the TF scaling parameters $\lambda_{z}(t)$ and $\lambda_{x}(t)$ we define

$$
\begin{aligned}
& \lambda_{z}(t)=\sqrt{\frac{\left\langle z^{2}(t)\right\rangle}{\left\langle z^{2}(0)\right\rangle}}, \\
& \lambda_{x}(t)=\sqrt{\frac{\left\langle x^{2}(t)\right\rangle}{\left\langle x^{2}(0)\right\rangle}} .
\end{aligned}
$$

Furthermore, the total energy $E_{\text {tot }}$ of the dipolar BEC is numerically evaluated from the GPE energy functional [41] and given by

$$
E_{\text {tot }}=\int_{V}\left(\frac{\hbar^{2}}{2 m}|\nabla \psi|^{2}+V_{\text {ext }}|\psi|^{2}+\Phi_{d d}|\psi|^{2}+\frac{g}{2}|\psi|^{4}\right) .
$$

The terms in the integrand correspond to, from left to right, the kinetic energy $E_{k}$, potential energy $E_{p}$, dipolar interaction energy $E_{d}$, and $s$-wave interaction energy $E_{s}$. Note that the zero-point kinetic energy is the kinetic energy associated with variations in the real part of $\psi$. Expressing $\psi=\psi_{r}+i \psi_{i}$, where $\psi_{r}$ and $\psi_{i}$ are real quantities, the $|\nabla \psi|^{2}$-term in Eq. (B5) can be decomposed as $|\nabla \psi|^{2}=\left[\nabla \psi_{r}\right]^{2}+\left[\nabla \psi_{i}\right]^{2}$. The zeropoint kinetic energy can thus be defined as

$$
E_{\mathrm{zp}}=\int_{V} \frac{\hbar^{2}}{2 m}\left[\nabla \psi_{r}\right]^{2} \text {. }
$$

[1] P. A. Robinson, Rev. Mod. Phys. 69, 507 (1997).

[2] Y. S. Kivshar and D. E. Pelinovsky, Phys. Rep. 331, 117 (2000).

[3] A. Y. Wong and P. Y. Cheung, Phys. Rev. Lett. 52, 1222 (1984).

[4] C. C. Bradley, C. A. Sackett, and R. G. Hulet, Phys. Rev. Lett. 78, 985 (1997).

[5] J. M. Gerton, D. Strekalov, I. Prodan, and R. G. Hulet, Nature (London) 408, 692 (2000)

[6] J. L. Roberts, N. R. Claussen, S. L. Cornish, E. A. Donley, E. A. Cornell, and C. E. Wieman, Phys. Rev. Lett. 86, 4211 (2001).

[7] J. K. Chin, J. M. Vogels, and W. Ketterle, Phys. Rev. Lett. 90, 160405 (2003).

[8] T. Koch et al., Nat. Phys. 4, 218 (2008).

[9] T. Lahaye, J. Metz, B. Frohlich, T. Koch, M. Meister, A. Griesmaier, T. Pfau, H. Saito, Y. Kawaguchi, and M. Ueda, Phys. Rev. Lett. 101, 080401 (2008).

[10] C. A. Sackett, H. T. C. Stoof, and R. G. Hulet, Phys. Rev. Lett. 80, 2031 (1998).

[11] A. Gammal, T. Frederico, and L. Tomio, Phys. Rev. A 64, 055602 (2001).

[12] N. G. Parker, S. L. Cornish, C. S. Adams, and A. M. Martin, J. Phys. B 40, 3127 (2007).

[13] K. Góral, K. Rzażewski, and T. Pfau, Phys. Rev. A 61, 051601(R) (2000).

[14] S. Yi and L. You, Phys. Rev. A 61, 041604(R) (2000).

[15] L. Santos, G. V. Shlyapnikov, P. Zoller, and M. Lewenstein, Phys. Rev. Lett. 85, 1791 (2000).

[16] S. Yi and L. You, Phys. Rev. A 63, 053607 (2001).

[17] K. Góral and L. Santos, Phys. Rev. A 66, 023613 (2002).

[18] C. Ticknor, N. G. Parker, A. Melatos, S. L. Cornish, D. H. J. O'Dell, and A. M. Martin, Phys. Rev. A 78, 061607(R) (2008).

[19] J. L. Bohn, R. M. Wilson, and S. Ronen, e-print arXiv:0809.4727v1.

[20] D. H. J. O’Dell, S. Giovanazzi, and G. Kurizki, Phys. Rev. Lett. 90, 110402 (2003).

[21] L. Santos, G. V. Shlyapnikov, and M. Lewenstein, Phys. Rev. Lett. 90, 250403 (2003).

[22] S. Giovanazzi and D. O’Dell, Eur. Phys. J. D 31, 439 (2004).
[23] U. R. Fischer, Phys. Rev. A 73, 031602(R) (2006).

[24] S. Ronen, D. C. E. Bortolotti, and J. L. Bohn, Phys. Rev. Lett. 98, 030406 (2007).

[25] R. M. Wilson, S. Ronen, J. L. Bohn, and H. Pu, Phys. Rev. Lett. 100, 245302 (2008).

[26] S. Giovanazzi, D. H. J. O'Dell, and G. Kurizki, Phys. Rev. Lett. 88, 130402 (2002).

[27] O. Dutta, R. Kanamoto, and P. Meystre, Phys. Rev. Lett. 99, 110404 (2007).

[28] O. Dutta and P. Meystre, Phys. Rev. A 75, 053604 (2007).

[29] A. Griesmaier, J. Werner, S. Hensler, J. Stuhler, and T. Pfau, Phys. Rev. Lett. 94, 160401 (2005).

[30] Q. Beaufils, R. Chicireanu, T. Zanon, B. Laburthe-Tolra, E. Marechal, L. Vernac, J. C. Keller, and O. Gorceix, Phys. Rev. A 77, 061601(R) (2008).

[31] K.-K. Ni, et al., Science 322, 231 (2008).

[32] The definition $\varepsilon_{d d}$ arises naturally from an analysis of the frequencies of collective excitations in a homogeneous dipolar BEC: for $\varepsilon_{d d}>1$ instabilities can occur in the hydrodynamic limit [13].

[33] S. Giovanazzi, A. Görlitz, and T. Pfau, Phys. Rev. Lett. 89, 130401 (2002).

[34] D. H. J. O’Dell, S. Giovanazzi, and C. Eberlein, Phys. Rev. Lett. 92, 250401 (2004).

[35] C. Eberlein, S. Giovanazzi, and D. H. J. O’Dell, Phys. Rev. A 71, 033618 (2005).

[36] N. G. Parker and D. H. J. O’Dell, Phys. Rev. A 78, 041601(R) (2008).

[37] S. Ronen, D. C. E. Bortolotti, and J. L. Bohn, Phys. Rev. A 74, 013623 (2006).

[38] E. Madelung, Z. Phys. 40, 322 (1927).

[39] Yu. Kagan, E. L. Surkov, and G. V. Shlyapnikov, Phys. Rev. A 54, R1753 (1996); 55, R18 (1997).

[40] Y. Castin and R. Dum, Phys. Rev. Lett. 77, 5315 (1996).

[41] F. Dalfovo, S. Giorgini, L. P. Pitaevskii, and S. Stringari, Rev. Mod. Phys. 71, 463 (1999).

[42] S. Giovanazzi, P. Pedri, L. Santos, A. Griesmaier, M. Fattori, T. Koch, J. Stuhler, and T. Pfau, Phys. Rev. A 74, 013621 (2006).

[43] E. Zaremba, Phys. Rev. A 57, 518 (1998).

[44] T. Lahaye et al., Nature (London) 448, 672 (2007). 